\title{
The VIL gene CRAWLING ELEPHANT controls maturation and differentiation in tomato via polycomb silencing
}

Ido Shwartz ${ }^{1}$, Chen Yahav ${ }^{1}$, Neta Kovetz ${ }^{1}$ Alon Israeli ${ }^{1}$, Maya Bar ${ }^{1,6}$, Matan Levy ${ }^{1}$, Katherine L. Duval ${ }^{2,5}$, José M. Jiménez-Gómez ${ }^{3,4}$ Roger B. Deal $^{2}$ and Naomi Ori ${ }^{1 *}$

anstitute of Plant Sciences and Genetics in Agriculture, The Robert H. Smith Faculty of Agriculture, Food and Environment, The Hebrew University of Jerusalem, Rehovot 76100, Israel; ${ }^{2}$ Emory University, Department of Biology, O. Wayne Rollins Research Center, 1510 Clifton Road NE, Atlanta , GA 30322; ${ }^{3}$ Department of Plant Breeding and Genetics, Max Planck Institute for Plant Breeding Research, Cologne, Germany; ${ }^{4}$ Institut Jean-Pierre Bourgin, INRAE, AgroParisTech, Université ParisSaclay, 78000, Versailles, France;

${ }^{5}$ Current address: University of Georgia, Department of Genetics, Athens, Georgia 30602

${ }^{6}$ Current address: Department of Plant Pathology and Weed Research, ARO, Volcani Center, Bet Dagan, 75101 Israel

*Corresponding author, naomi.ori@mail.huji.ac.il 


\begin{abstract}
:
VERNALIZATION INSENSITIVE 3-LIKE (VIL) proteins are PHD-finger proteins that recruit the repressor complex Polycomb Repressive Complex 2 (PRC2) to the promoters of target genes. Most known VIL targets are flowering repressor genes. Here, we show that the tomato VIL gene CRAWLING ELEPHANT (CREL) promotes differentiation throughout plant development by facilitating the trimethylation of Histone H3 on lysine 27 (H3K27me3). We identified the crel mutant in a screen for suppressors of the simple-leaf phenotype of entire (e), a mutant in the AUX/IAA gene ENTIRE/SIIAA9, involved in compound-leaf development in tomato. crel mutants have increased leaf complexity, and suppress the ectopic blade growth of $e$ mutants. In addition, crel mutants are late flowering, and have delayed and aberrant stem, root and flower development. Consistent with a role for CREL in recruiting PRC2, crel mutants present altered $\mathrm{H} 3 \mathrm{~K} 27 \mathrm{me} 3$ modifications at a subset of PRC2 targets throughout the genome. Our results uncover a wide role for CREL in plant and organ differentiation in tomato and suggest that CREL is required for targeting PRC2 activity to, and thus silencing, a specific subset of polycomb targets.
\end{abstract}

\title{
Author summary:
}

Plants form organs continuously throughout their lives, and the number and shape of their organs is determined in a flexible manner according to the internal and external circumstances. Alongside this flexibility, plants maintain basic developmental programs to ensure proper functioning. Among the ways by which plants achieve flexible development is by tuning the pace of their maturation and differentiation, at both the plant and organ levels. One of the ways plants regulate the rate of maturation and differentiation is by changing gene expression. Here, we identified a gene that promotes plant and organ maturation and differentiation. This gene, CRAWLING ELEPHANT (CREL) acts by bringing a repressing complex to target genes. We show the importance of CREL in multiple developmental processes and in the expression of multiple genes throughout the tomato genome.

\section{Introduction:}

Polycomb Repressive Complex 2 (PRC2) is a conserved complex that represses gene expression by trimethylating lysine 27 of histone $\mathrm{H} 3$ proteins (H3K27me3)[1-3]. PRC2 
activity counteracts, and is counteracted by, the transcription-promoting functions of trithorax-group proteins [4]. The core PRC2 is composed of 4 subunits. In plants, some of these subunits are encoded by small gene families, allowing the formation of multiple, distinct complexes. Different plant PRC2 complexes have been shown to regulate specific developmental processes such as endosperm development, flowering time and flower development [2,3]. As PRC2 complexes do not have DNA binding domains, they are recruited to target loci by interacting proteins [2,5-7]. One of the most characterized PRC2-regulated processes in Arabidopsis is the induction of flowering in response to prolonged cold, termed vernalization. In response to vernalization, PRC2 promotes flowering by silencing the flowering inhibitor FLC. The vernalization-specific VRN-PRC2 complex is recruited to FLC by complexing with PHD proteins from the VERNALIZATION INSENSITIVE 3-LIKE (VIL) family [710]. In Arabidopsis, the VIL family consists of 4 members, including VIN3 and VRN5. Vernalization induces VIN3 expression, while VRN5 is expressed constitutively. VIL proteins also repress additional members of the FLC family during vernalization, and VRN5 and VIL2 are also involved in other flowering pathways $[7,8,11,12]$.

VIL proteins have been identified from several plant species [13-22]. They have been shown to promote flowering in all tested species, including species that do not have an FLC ortholog and/or do not respond to vernalization. In rice, the OsLF and OsLFL1 genes encode transcription factors that inhibit flowering and have been identified as VIL targets [15,17]. A VIN3 ortholog has also been identified in tomato [21]. While the vast majority of research on VIL proteins concerned their involvement in flowering induction, several studies reported additional developmental effects. For example, Arabidopsis vrn5 mutants had increased leaf curling, increased numbers of petals, and distorted siliques [10]. In rice, osvil3/leaf inclination2 (lc2) mutants had an altered leaf angle, curled leaves and severe sterility, and OsVIL2 was found to affect spikelet development, branching and grain yield [13,14,16,23]. Silencing the Brachypodium distachyon BdVIL4, which is similar to VIN3, led to increased branching [18]. Pepper cavill mutants affect leaf development, apical dominance and branching [22]. However, the knowledge about the involvement of VIL proteins in these and other developmental processes is limited, and their role in compound-leaf development has not been explored. In addition, it is not clear whether VIL proteins recruit PRC2 mainly 
to targets involved in the induction to flowering or whether they have broader roles in plant development.

Tomato plants have compound leaves, which are composed of multiple leaflets [24]. The elaboration of compound leaves depends on slow maturation of the developing leaf, which enables an extended organogenesis activity at the leaf margin, during which leaflets are formed [25-29]. Leaflets are formed by differential growth at the leaf margin, where regions of blade growth are separated by intercalary regions of growth inhibition [30]. Auxin has been shown to promote growth and its response is inhibited in the intercalary domains [31-37]. Mutations in the tomato gene SIIAA9/ENTIRE (E), encoding an auxin-response inhibitor from the Aux/IAA family that specifies the intercalary domain, result in simplified leaves due to ectopic blade growth in the intercalary domain $[31,38,39]$.

Here, a screen for suppressors of the $e$ simple-leaf phenotype identified the crawling elephant ( $\mathrm{crel}$ ) mutant, which substantially suppresses the ectopic blade growth of $e$. We found CREL to encode a tomato VIL gene, related to Arabidopsis VILI/VRN5. crel mutants affect many aspects of tomato development, including plant and organ maturation. Comparison of $\mathrm{H} 3 \mathrm{~K} 27 \mathrm{me} 3$ modifications between wild type and crel plants showed that CREL affects these modifications at some PRC2 targets and not others. Therefore, CREL promotes maturation throughout the plant life by promoting selective deposition of $\mathrm{H} 3 \mathrm{~K} 27 \mathrm{me} 3$ and gene silencing at a subset of PRC2 targets.

\section{Results:}

crawling elephant (crel) mutants suppress entire (e) and have very compound leaves

entire (e) mutants, mutated in a tomato Aux/IAA gene, have simplified leaves in comparison to the wild-type compound leaves [31,37,39,40] (Fig 1A, B). To identify genes that are involved in compound-leaf development, we generated an Ethyl Methane Sulfonate (EMS) mutant population in the background of $e$, and screened for suppressors of the $e$ simplified leaf phenotype. This screen identified the crawling elephant-1 (crel-1) mutant as a strong e suppressor. e crel-1 double mutants had distinct, clearly separate primary leaflets, and occasionally had secondary leaflets, in contrast to the mostly entire leaf shape of single $e$ mutants (Fig 1A-C). To characterize the unique crel-1 phenotype, we backcrossed crel-1 to the parental line (Solanum 
lycopersicum M82), and identified single crel-1 F2 individuals (Fig 1D). Leaves of single crel-1 mutants were much more compound than wild-type leaves, with a similar number of primary leaflets but many more secondary leaflets than the wild type. In contrast to wild type-leaves, crel-1 leaves also had tertiary leaflets (Fig 1A, D, J, K). Therefore, crel mutants suppress the $e$ simplified-leaf phenotype, and forms many more leaflets in both the wild type and the $e$ backgrounds. Interestingly, previously identified $e$ suppressors such as $\operatorname{sim} p$ and slarf19a,b had a reduced number of leaflets in the respective single mutants [36].

We identified several additional crel alleles from the Menda EMS and fast neutron mutant population [41], in the M82 background, and confirmed allelism by complementation tests. The alleles showed a range of phenotypic severities, including a diverse increase in leaflet number (Fig 1E-H, J, K). Similar to crel-1, crel-2 also suppressed the $e$ simplified leaf phenotype (S1 Fig A-D).

\section{CREL acts during relatively late stages of leaf development:}

To investigate the timing of the effect of crel mutants on leaf development, we compared leaf development between wild type and crel-1 plants. Early stages of leaf development were very similar between wild type and crel-1 plants when similar developmental stages were compared, although the terminal leaflet expanded earlier in crel-1 (Fig 1I). However, the rate of leaf and leaflet initiation was much slower in crel1 mutants than in the wild type (S1 Fig E). At later stages of leaf development, when wild type leaves stopped generating new leaflets, $\mathrm{crel}-1$ and $\mathrm{crel}-2$ leaves continued to form leaflets more than a month later (Fig 1K). Therefore, crel leaves develop slower than the wild type, and while the terminal leaflet appears to differentiate early, overall leaf differentiation is substantially delayed in crel mutants.

To further characterize this effect of crel on leaf development, and understand the timing and developmental context of CREL action, we analyzed its genetic interaction with mutants that affect the developmental window of leaflet morphogenesis. Leaflets are formed during the morphogenesis stage of leaf development, which follows leaf initiation and precedes leaf expansion and differentiation [24,25,42,43]. The elaboration of compound leaves depends on an extended morphogenesis stage. The CIN-TCP transcription factor LANCEOLATE (LA) and the MYB transcription factor CLAUSA (CLAU) promote maturation and differentiation and thereby restrict the 
morphogenetic window [26,44-47]. La-2 is a semi-dominant mutant in which $L A$ is expressed precociously due to a mutation in the miR319 binding site. This accelerates leaf differentiation and leads to a simple leaf form (Fig 2A, C). La-2 was epistatic to crel-1 (Fig 2 B-D), indicating that the morphogenetic window in La-2 is terminated before the timing of CREL action, in agreement with the relatively early effect of $L A$ and late effect of CREL on leaf development. Leaves of loss-of-function clau mutants have an extended morphogenetic window, leading to a substantial increase in leaf complexity and leaflet number (Fig 2E) $[45,48]$. crel-1 clau double mutants had very complex leaves (Fig 2E, F), suggesting that CREL acts in parallel with CLAU to restrict leaf elaboration and promote maturation. Removing the activities of both regulators leads to prolonged, extensive leaflet morphogenesis. Together, these results suggest that CREL acts in relatively late stages of leaf development to promote maturation and differentiation.

The suppression of the $e$ phenotype by crel raised the question of whether CREL is also involved in the differential growth at the leaf margin that leads to the formation of separate leaflets. To address this question, we crossed crel to mutants affected in auxinmediated blade growth. Ectopic expression of a stabilized form of E resulting from a mutation in domain II of the E (IAA9) protein (EdII) resulted in leaflet narrowing [39], (Fig 2G). This effect was strongly enhanced in the crel-1 background (Fig 2H), suggesting that $\mathrm{E}$ inhibits and CREL promotes blade expansion, but they act in at least partially parallel pathways. Similarly, crel-1 enhanced the narrow blade phenotype resulting from ectopic expression of a miR160-resistant ARF10, a negative regulator of blade expansion ( FIL >>ARF10m, Fig 2I, J). In agreement, crel-1 suppressed the ectopic blade growth resulting from ectopic expression of miR160, which negatively regulates ARF10 and 4 additional ARF proteins (FIL>>miR160, Fig 2K, L) [37]. The suppression of $F I L>>m i R 160$ was more prominent in later leaves than in early leaves. Interestingly, leaflet number was reduced in $F I L>>A R F 10$ crel- 1 relative to both single mutants, and crel-1 FIL> $>$ EdII-GUS plants were extremely small with almost no leaflets, suggesting that extreme repression of lamina growth leads to a reduction in leaflet formation and overall growth. Together, these results suggest that CREL promotes blade growth during compound-leaf development, and acts at least partially in parallel to auxin. 


\section{CREL is a VRN5 homolog:}

To identify the CREL gene, we genetically mapped the crel-1 mutation using an F2 mapping population from a cross between the crel-1 mutant, in the Solanum lycopersicum M82 background, and S. pimpinellifolium. crel-1 was mapped to the short arm of chromosome 5. Further mapping was hampered by an introgression of $S$. pimpinellifolium sequences in the M82 line in this region [49]. We therefore used RNAseq to identify possible causative mutations in crel-1 and crel-2, which led to the identification of mutations in the gene Solyc05g018390 in both crel-1 and crel-2. In crel-1, a G to A substitution at position 4264 from the transcription start site (TSS) led to a stop codon in exon III. The fast neutron allele crel-2 contains a 12,826-bp-long deletion, which results in the elimination of exon I and II and part of exon III (Fig 3A). Sequencing the Solyc05g018390 gene in two additional crel alleles identified a 1-bp deletion in the first exon at position 322 from the TSS in crel-3, and an A to T substitution in position 3630 leading to a stop codon in the third exon in crel-5 (Fig 3A). We therefore concluded that Solyc05g018390 is CREL. CREL is predicted to encode a plant homeodomain (PHD) finger protein (Fig 3A, B). It is most similar to the Arabidopsis VRN5 gene.

\section{CREL is expressed in expanding blades:}

We characterized the expression of $C R E L$ in the fifth leaf produced by the plant, at different developmental stages, to examine how its expression correlates with its activity. CREL was expressed throughout leaf development, with relatively low expression in apices containing the SAM and very young P1-P3 primordia. Later, its expression was gradually upregulated, peaking at P6/P7 (Fig 3C). To spatially localize CREL in leaf primordia, we cloned a 2960-long CREL promoter and used it to generate a CREL driver line in the transactivation system [50,51]. In developing leaves, the CREL promoter drove expression in leaf margins. In agreement with the qPCR experiment, expression appeared lower in young primordia, and increased from $\mathrm{P} 4$ on. Expression was mainly visible in expanding regions of the leaf margin, the terminal leaflet at P4, and the expanding leaflets at P6 and on (Fig 3D-G). The expression appeared to follow the basipetal differentiation wave of the leaf, with strong expression first appearing in the terminal leaflet, which is the first to expand and differentiate, and then progressing basipetally in expanding leaflets. This leaf expression pattern is compatible with the crel leaf phenotype, which starts to differ from the wild type around 
the P5 stage (Fig 1I), and with the genetic interactions showing that crel affects leaf maturation and blade formation (Fig 2).

\section{CREL promotes multiple aspects of plant maturation and differentiation:}

In addition to their effect on leaf differentiation and patterning, additional differentiation processes were also delayed and/or impaired in crel mutants. crel plants failed to maintain an upright position and the plants exhibited a sprawling growth habit. Similar to the effect on leaf shape, this phenotype developed at a relatively late stage of plant development (Fig 4A, B). To further understand the role of CREL in plant development, we overexpressed the CREL gene under the control of the $35 \mathrm{~S}$ promoter. CREL mRNA expression increased only slightly in 35S:CREL plants (S2 Fig A), and the phenotypic effect was subtle (Fig 4A, B), but when mature plants were compared, 35S:CREL plants were slightly taller than crel-2 mutants (S2 Fig B). To understand the basis for the "crawling" phenotype, we dissected developing wild-type and crel-2 stems at successive developmental stages. We sectioned the internode between the cotyledons and the first leaf from different plants grown together, between the ages of 3 and 10 weeks. In three-week-old plants, crel-2 stems were narrower than the wild type with nearly normal although slightly less developed vascular bundles (Fig 4C, D, J, K). crel2 vasculature continued to develop slower than the wild type, and ceased maturation and differentiation prematurely. This resulted in a thin and undeveloped xylem in crel2 stems. Specifically, crel-2 stems failed to complete a vascular cylinder, and had only partial secondary xylem development (Fig 4E-H, L-O). The reduction in supporting tissue likely contributes to the reduced strength of the crel stem. Stem sections of 10week-old 35S:CREL plants were similar to those of the wild type but appeared to mature more slowly (Fig 4I, P).

Root vasculature development was also delayed and impaired in crel mutants. crel-2 roots were narrower than wild-type roots, and their vascular tissue developed slowly and failed to reach full differentiation (Fig 5A-F). To investigate the effect of crel on the root system as a whole, wild type and crel-2 plants were grown hydroponically, and root volume and length were calculated at successive times. Root volume and length were reduced in crel-2 plants, and the difference increased with time, although the difference was statistically significant at one of the time points only (Fig 5G,H). Therefore, CREL plays an important role in root development and differentiation. 
The $\mathrm{crel}$ mutation also affected flowering time and flower development. $\mathrm{crel}$ - 1 and $\mathrm{crel}$ 2 mutants flowered much later than the wild type, after producing 12-13 leaves, compared to 6 leaves in the wild type (Fig 6A). 35S:CREL plants flowered slightly earlier than the wild type, but this effect was statistically significant in only one of the lines (S2 Fig C). Mature crel flowers were not fully developed, had short and distorted organs and were sterile (Fig 6B, C). Early flower development was similar between wild type and crel plants, except for the sepals that were curled backwards in crel, resulting in an open bud where the inner organs were not covered by the sepals. However, crel flower organs ceased development and growth prematurely (Fig 6D-G). 35S:CREL flower development was similar to that of the wild type and the plants were fertile (data not shown). Therefore, crel mutants were delayed in multiple developmental pathways. In some cases such as flower, stem, and root development, these organs failed to properly differentiate, while in others, such as leaf development and flowering time, they differentiated substantially slower than the wild type. Overall, crel plants had aberrant plant and organ structure, which resulted in weak and sterile plants.

\section{CREL mediates H3K27me3 modifications at a subset of polycomb-silenced genes:}

Homology of CREL to the Arabidopsis VRN5 gene suggested that it may be involved in the repression of gene expression by promoting PRC2-mediated H3K27me3 modification. To test this prediction, we performed ChIP-seq for the H3K27me3 modification in shoot apices of 4-week-old wild-type and crel-2 plants (Supplemental Table 1). In wild-type tissue, H3K27me3 was found to be significantly enriched at a total of 13,849 sites, mostly over gene bodies, as expected. In the crel-2 mutant, H3K27me3 appeared to be completely lost at 6,762 of these sites (48.8\%) normally enriched with H3K27me3 (Fig 7A), supporting the hypothesis that CREL normally guides deposition of $\mathrm{H} 3 \mathrm{~K} 27 \mathrm{me} 3$ at a subset of PRC2 target genes. Interestingly, 4,789 sites actually show significant increases in H3K27me3 in the crel-2 mutant (Fig 7A). The vast majority of these sites are normally enriched for H3K27me3 in WT, suggesting that in the absence of CREL, excess PRC2 activity is directed to the remaining target genes.

To examine the effects of these H3K27me3 re-distributions on target gene expression, we used reverse transcription followed by quantitative PCR (RT-qPCR) to examine the 
levels of several target transcripts in young leaf primordia. The tested genes were among the genes that were normally enriched for H3K27me3 in WT and lost modification in crel-2, and were upregulated in crel mutants in the RNAseq used for the identification of the CREL gene. We compared the expression of three such genes, GA20oxidase 3 (GA20ox3, Solyc11g072310), ARGONAUTE 5 (AGO5, Solyc06g074730) and PECTINESTERASE (Solyc02g080200), in P5 primordia of the fifth leaves produced by the plant. As expected, these genes showed increased transcript levels in the mutant (Fig 7B). These results are all consistent with the known role of H3K27me3 in gene silencing and further support the validity of our ChIP-seq data.

\section{Discussion:}

VIL proteins have been shown to affect flowering in several plant species, by repressing the expression of flowering repressors, such as FLC in Arabidopsis. In addition to their effect on flowering, VIL genes were found to affect an array of developmental processes in different species. This work identifies the tomato VIL gene CREL as a mediator of diverse developmental processes, via the modulation of $\mathrm{H} 3 \mathrm{~K} 27 \mathrm{me} 3$ modifications in many genes throughout the tomato genome, likely by recruiting PRC2 complexes to a subset of their target genes.

\section{CREL promotes plant and organ maturation:}

So far, VIL genes have been mainly implicated in flowering time $[7,11,13,15,17-$ $19,22,52]$. Here, we uncover a much broader role for this gene family in plant development, as revealed from the phenotypes and the effect on H3K27me3 modification. crel mutants are affected in many aspects of plant maturation and differentiation in addition to the delay in flowering time. crel mutants flower late and have delayed leaf maturation, resulting in an extended leaf morphogenesis and more compound leaves. Interestingly, while flowering and leaf maturation eventually occur in $\mathrm{crel}$, stem, root, and flower differentiation are impaired in crel and these organs do not reach full differentiation and function. CREL accumulates relatively late during leaf development, thus enabling prolonged morphogenesis. Recently, a growth-rate dependent mechanism of controlling VIN3 accumulation in the cold has been described [53]. It would be interesting to understand the mechanism by which CREL expression is delayed during organ maturation to enable timed maturation and differentiation. 
Other genes involved in the induction of flowering were also shown to affect maturation and differentiation in additional developmental aspects. For example, the tomato flowering inducer SFT, the ortholog of FT, was shown to promote leaf maturation and affect stem differentiation [54]. Recently, SFT was shown to specifically affect secondary cell wall biosynthesis in tomato stems [55]. FT was also proposed to promote maturation and termination in additional species [56,57]. The pepper cavill mutants, impaired in the pepper $C R E L$ ortholog, have reduced vascular development but wider stems [22]. Therefore, both precocious and delayed differentiation impairs the final form and function of stems. In Cardamine hirsuta, plant maturation and flowering was shown to be coordinated with age-dependent changes in leaf shape in plants with variable FLC activity [58]. In contrast to CREL and SFT, which promoted all aspects of plant and organ maturation, tomato CIN-TCPs were shown to promote leaf maturation but delay plant maturation, while AP1/FUL MADs BOX genes promoted plant maturation and delayed leaf maturation [59,60]. Overall, similar to CREL, genes that have been implicated mainly in the promotion of flowering in Arabidopsis were found to promote a wide range of differentiation and maturation aspects.

The involvement of CREL in plant and organ differentiation is in agreement with a role in mediating PRC2 activity. A common function of PRC2 genes in plants is the maintenance of a differentiated state, and prc2 mutants in both mosses and seed plants have phenotypes related to dedifferentiation and overproliferation [1,2,61]. Therefore, CREL may aid in recruiting PRC2 to differentiation-related target genes.

VIL genes from other species have also been shown to affect other developmental processes in addition to flowering. [10,13,16,18,22,23]. Interestingly, beside the common effect on flowering time, the specific developmental effects only very partially overlap among these species. This suggests that the VIL family may be used as a tool for developmental innovations, recruiting an existing tool to different processes. Specifically interesting in this respect is the comparison between tomato and pepper, which are closely related species that differ in several key developmental features, such as flowering architecture and leaf shape. cavill mutants have reduced vasculature development, increased plant and organ size, increased branching and reduced angle of axillary branches [22]. Interestingly, only some of these additional phenotypes overlap with $\mathrm{crel}$. In contrast to the simple leaves of pepper, tomato leaves are compound, with several orders of leaflets. This is correlated with faster differentiation of the pepper leaf, 
similar to tomato $L a-2 /+$ mutants [26]. The current work revealed an important role for CREL in the development of the compound leaf, with an effect on both the rate of differentiation and leaf patterning (Fig 1 and 2), further supporting the notion that VIL genes have been recruited to diverse, partially species-specific processes.

We propose that, in addition to its general effect on growth and differentiation, CREL also promotes blade growth in developing tomato leaves. crel mutants suppress the ectopic blade outgrowth of $e$ mutants and miR160-overexpressing plants (Fig 2K, L). Furthermore, crel enhances the narrow-leaf phenotype caused by overexpression of $E$ or miR160-targeted ARFs. In addition, CREL expression is elevated in later stages of leaf development when the blade begins to expand, and the CREL promoter shows high expression in growing regions of the leaf margin (Fig 3D-G)). The genetic interactions between crel and auxin-related mutants suggest these auxin mediators and CREL act via independent pathways to regulate blade growth. Therefore, CREL likely promotes blade growth either downstream of auxin or through an at least partially parallel pathway. As most effectors of compound leaf development have been shown to affect either the organ-level differentiation rate (for example LA and CLAU) or local differential growth (E, CUC, SIMP) [24], it is interesting that CREL appears to affect both aspects.

\section{CREL affects H3K27me3 modifications throughout the tomato genome:}

Only a handful of VIL targets have been identified so far, most of which are related to their role in promoting flowering. In Arabidopsis, FLC and FLC-related genes are targeted by different VIL protein in specific flowering pathways [8,11]. In rice, the flowering inhibitors $O s L F$ and $O s L F L 1$ were identified as VIL targets $[15,19,62]$. In addition, a cytokinin catabolism gene from the CKX family and the bud-outgrowth inhibitor OsTB1 have been identified as a VIL target in rice [16,23]. The microRNA miR156 was proposed as a target of BdVIL4 in Brachypodium [18], and several putative targets have been proposed to mediate the effect on flowering of pepper VIL1 [22]. The identification of a global effect on $\mathrm{H} 3 \mathrm{~K} 27 \mathrm{me} 3$ modification in crel mutants suggests that there are many more VIL targets than previously described. Together with the pleiotropic phenotypic effect, this suggests that VIL proteins are involved in a wide range of developmental processes, and play a central role in recruiting PRC2 complexes to many targets genome wide. The similarly pleiotropic effect of Cavill mutants in pepper, together with its effect on gene expression [22], suggests that this is also true in 
other species.

\section{A conserved role for VIL genes in promoting flowering:}

VIL genes have been shown to affect flowering time in many species where mutations or silencing of these genes have been described, including both dicots and monocots [7,11,13,15,17,19,22]. In Arabidopsis, VIL proteins promote flowering by recruiting PRC2 to flowering repressors from the FLC family, thus facilitating the deposition of the repressive chromatin modification H3K27me3. Different Arabidopsis VILs act to induce flowering in specific combinations of flowering pathways, timing and target genes [8,11]. Interestingly, while tomato plants do not require vernalization for flowering and also lack $F L C$, crel mutants are late flowering. Similarly, VIL genes promote flowering in additional species that do not require vernalization for flowering and do not have a close homolog of FLC, or in species with a different vernalization mechanism. In rice, OsVIL2 and OsVIL3/LC were shown to act by repressing OSLFL1 and $O s L F$, respectively, two flowering repressors unrelated to FLC $[15,19,62]$. Therefore, while the effect on flowering and possibly the molecular mechanism are conserved, the target genes differ among species [22]. It will be interesting to identify the flowering repressor that mediates this effect in tomato.

\section{Materials and methods}

\section{Plant material and growth conditions}

Tomato plants (Solanum lycopersicum cv M82) were germinated and grown in a controlled growth room or in a commercial nursery for four weeks. Then the seedlings were transferred to a greenhouse with natural daylight and $25^{\circ} \mathrm{C} / 20^{\circ} \mathrm{C}$ day/night temperature, or to an open field with natural daylight and temperature. crel-1 was isolated in this work by a mutant screen in the $e-3$ background (Berger 2009, Ben Gera 2012), as described below. crel-2 - crel-5 are from the mutant populations described by Menda et al.,[41]. The transactivation system, described previously [50,51], was used to characterize the CREL promoter and for leaf-specific expression. This system consists of driver lines and responder lines. In the driver lines, specific promoters drive the expression of the synthetic transcription factor LhG4, which does not recognize endogenous plant promoters. In the responder lines, a gene of interest or a reporter is expressed downstream of the E.coli operator, recognized by LhG4 but not endogenous plant transcription factors. A cross between a driver and a responder lines results in a 
plant (designated PROMOTER>>GENE) expressing the gene of interest/marker under the control of the specific promoter. La-2, clau, the FIL driver line and the ARF10, miRl60 and EdII-GUS responder lines have been previously described $[32,37,44,48,51,63]$. 35S::CREL lines were generated in this work, as described below.

\section{Generation of a mutant population, screening and identification of $\mathrm{crel}-1$}

Around 750 entire-4 (e-4) seeds were treated with the mutagenic substance EthylMethane Sulfonate (EMS, Sigma m0880) at a concentration of $0.6 \%$ for 10 hours. Around 50 seeds underwent a control treatment without exposure to EMS. The treated seeds were sown in a commercial nursery and the seedlings (M1 generation) were transferred to a greenhouse. M1 plants were self-pollinated to increase the number of seeds per plant. M2 seeds were collected separately from each of around $650 \mathrm{M} 1$ plants. M2 progeny (around 40 seeds per family) were grown in an open field, and screened frequently during the season for mutants that affect the development of the leaf, flower and fruit. crel-1 was identified in this screen as a recessive mutant segregating 1:3 in an M1 family. Single crel-1 mutants were generated by a cross between $e$ crel-1 and wild type and identification of single crel-1 mutants in the F2 generation. crel-1 was then back-crossed three times to M82 for further characterization.

\section{Allelism tests and genetic interactions:}

As crel mutants are sterile, allelism tests were performed by crossing heterozygote siblings. Progeny of a cross between two heterozygous alleles segregated 1/4 mutant progeny. Similarly, genetic interactions between $\mathrm{crel}$ and other mutants or transgenic lines were generated by crossing heterozygous $\mathrm{crel}$ siblings with the respective mutant or transgenic line.

\section{Identification of the $C R E L$ gene:}

An F2 mapping population was generated by crossing crel-1/+ plant, in the M82 background to Solanum pimplinellifolium, and collection of F2 progeny from individual F1 plants. Initial mapping with $30 \mathrm{~F} 2$ plants showing the crel-1 phenotype, and 50 mapping markers developed by Revital Bronstein, Yuval Eshed (Weizmann Institute) and Zach Lippman (CSHL) and spread along the tomato genome, identified linkage to 3 markers on chromosome 5. Fine mapping of 120 crel- 1 F2 individuals and additional markers located the gene to a region between markers zach 43.2 and jose 58.1 dcap, located between bases 43,123,344 and 58,170,500 on chromosome 5. Further mapping 
was hampered by an introgression of S. pimpinellifolium sequences in the M82 line in this region [49] We therefore used RNAseq of wild type, crel-1 and crel-2 plants to identify polymorphism in these alleles.

For RNAseq, shoot apices containing the SAM and the 4 youngest primordia were collected from 14-day-old M82, crel-1 and crel-2 plants, in which L4 (the $4^{\text {th }}$ leaf produced by the plant) was at the P4 stage. RNA was extracted using the RNeasy micro $\mathrm{kit}^{\mathrm{TM}}$ (Qiagen), using the manufacturer's instructions. Two biological replicates were used for M82 and crel-2, and one biological replicate for crel-1. Sequencing libraries were prepared according to the Illumina TruSeq RNA protocol and sequenced on an Illumina HiSeq2000 platform at the Genome Center of the Max Planck Institute for Plant Breeding Research. We obtained between 21,3 and 28,3 million 96-bp single-end reads per library (average of 25,8 million). Reads were aligned to the S. lycopersicum reference sequence v2.40 using TopHat v2.0.6 [64] with the following parameters: max-insertion-length 12 -max-deletion-length 12 -g 1 -read-gap-length 12 -read-editdist 20 -read-mismatches 12 -no-coverage-search -read-realign-edit-dist 0 -segmentmismatches 3 -splice-mismatches 1 . To detect polymorphisms between the $\mathrm{crel}$ mutants and wild-type M82, biological replicates from each genotype were merged. Then, duplicated reads were removed using default settings in Picard (http:// broadinstitute.github.io/picard/), indels were realigned using GATK v2.2-8, and variants called in all samples simultaneously using default parameters in GATK v2.28 [65]. Next, we estimated the effect of each variant in annotated transcripts (ITAG 2.3) using ANNOVAR [66]. Variants in the candidate region in chromosome 5 determined by QTL analysis were evaluated manually.

\section{Phenotyping and imaging:}

Characterization of early leaf development and rate of leaf initiation - Plants were sown, germinated and grown in a growth chamber. Every two weeks, the number of leaves and leaf primordia were counted from six plants from each genotype. The fifth leaf (L5) was photographed by a stereoscope (Nikon SMZ1270) and its developmental stage determined. Six different plants were used for each time point.

Quantification of leaf complexity - Leaves 5, 7 and 9 were marked at the time of their emergence from the shoot apex. Then, the number of leaflets was counted every 7-14 days for each marked leaf. Primary, intercalary, secondary and tertiary leaflets were 
counted. At least 9 plants were counted for each genotype.

SEM characterization of flower development - Flowers from different developmental stages of each genotype were collected and their petals removed using a stereoscope, placed on a microscope stub with a carbon strip and analyzed with Hitachi TM3030 Plus SEM.

Phenotypic quantification and statistical analysis - For the quantification of the number of leaves to flowering, plants were grown in a greenhouse, and with the appearance of the first flower, the number of leaves formed before the flower were counted. At least 9 biological repeats, each consisting of a single plant, were quantified. The experiment was repeated twice, once with plants germinated in a commercial nursery and once with plants germinated in a growth chamber. Quantification of plant height and width was performed on 15-week-old plants grown in a greenhouse. Plant height was measured from the soil to the stem tip. For the quantification of root phenotypes, seedlings were grown hydroponically in Hoagland nutrient solution $(\mathrm{pH}$ 6.5), in a growth room set to a photoperiod of 12/12-h night/days, light intensity (coolwhite bulbs) of $\sim 250 \mu \mathrm{mol} \mathrm{m} \mathrm{m}^{-2}$, and $25^{\circ} \mathrm{C}$. After $28,34,38$ and $43 \mathrm{DAG}$ the roots of 3 plants of each genotype were scanned and analyzed using a flatbed scanner (Epson 12000XL, Seiko Epson, Japan) and root architecture was analysed using WinRhizo software (Regent Instruments Ltd., Ontario,Canada). Statistical analysis was performed using the JMP software (SAS Institute, http://www.sas.com). Means and p values were calculated using the Student's t-test or the Dunnett's test, as indicated in the figures.

Histological characterization of stem tissues - Ten to 50 day-old plants were freehand dissected using a double-sided razor blade. 1-2-mm-long sections were dissected from up to $5 \mathrm{~cm}$ below the node. Sections were dehydrated in acetic acid: ethanol [1:10] for 1 hour and then stained directly with TBO (0.01\% aqueous, sigma). Images of early developmental stages were captured using Nikon a SMZ1270 stereoscope equipped with a Nikon DS-Ri2 camera and NIS-ELEMENTS software.

Confocal imaging - For analysis of pCREL:nYFP expression, dissected whole-leaf primordia were placed into drops of water on glass microscope slides and covered with cover slips. The pattern of YFP expression was detected by a confocal laser scanning microscope (CLSMmodel SP8; Leica), with the solid-state laser set at $514 \mathrm{~nm}$ for excitation and $530 \mathrm{~nm}$ for emission. Chlorophyll expression was detected at 488nm for 
excitation and 700nm for emission. ImageJ software was used for analysis, quantification, and measurements of captured images. Images were adjusted uniformly using Adobe Photoshop CS6. Tomato stems and primary roots were cut to sections of 200um and 300um width respectively using Leica VT1000 vibratome and were and cleared using ClearSee [67], cell wall staining was performed using SR2200 (Renaissance Chemicals) prior to mounting and visualization using 405nm laser.

\section{Cloning and plant transformation:}

The CREL promoter was generated by amplifying $3000 \mathrm{bp}$ upstream of the CREL ATG from genomic DNA and cloned upstream to LhG4 generating the $p C R E L: L h G 4$ driver line.

The CREL gene was amplified from tomato M82 cDNA using the op:VIN3 $\mathrm{F}$ and op:VIN3 R primers (S2 Table), and cloned into the $\mathrm{pENTR} / \mathrm{d}^{\mathrm{TM}}$ vector using a TOPO isomerase cloning system (Invitrogen). The CREL gene was then subcloned using L/R clonase (Invitrogen) downstream to the $35 \mathrm{~S}$ promoter to generate $35 S:: C R E L$.

Plant transformation and tissue culture were performed as described in Israeli et al 2019 [36]. At least five independent kanamycin-resistant transgenic lines from each transgene were genotyped and, in the case of $p C R E L: L h G 4$, crossed to an OP:YFP stable line to generate $\mathrm{pCREL}>>$ YFP. Three lines from each transgene or resultant cross were examined, and a representative line was selected for further analysis.

\section{Phylogenetic Analysis}

Phylogenetic analysis was performed using full-length protein sequences of the tomato, Arabidopsis, rice and pepper VIL gene family. The sequences were obtained from the Sol Genomics Network (SOL, https://solgenomics.net/), The Arabidopsis Information Resource (TAIR, https://www.arabidopsis.org/) and the Plaza tool (https://bioinformatics.psb.ugent.be/plaza/). Sequences were aligned and phylogenetic tree was constructed using Clustal W (https://www.ebi.ac.uk/Tools/msa/clustalo/) $[68,69]$.

\section{RNA extraction and $q R T-P C R$ analysis}

RNA was extracted using the Plant/Fungi Total RNA Purification Kit (Norgen Biotek, Thorold, ON, Canada) according to the manufacturer's instructions, including DNase treatment. cDNA synthesis was performed using the Verso cDNA Kit (Thermo 
Scientific, Waltham, MA, USA) using $1 \mu \mathrm{g}$ of RNA. qRT-PCR analysis was carried out using a Corbett Rotor-Gene 6000 real-time PCR machine, with SYBR Premix for all other genes. Levels of mRNA were calculated relative to EXPRESSED (EXP) [70] or TUBULIN (TUB) [71] as described [26]. Primers used for the qRT-PCR analysis are detailed in $\mathrm{S} 2$ table.

\section{ChIP-seq procedures}

WT and crel plants were grown on soil under $16 \mathrm{hrs}$ of light/ $8 \mathrm{hrs}$ dark cycles for 28 days after germination. Shoot apices, $(0.8 \mathrm{~g}$ for each replicate and two replicates per genotype) containing approximately three visible expanding leaves, were harvested and fixed in $1 \%$ formaldehyde $+0.2 \%$ Silwet L-77 for 17 minutes under vacuum. Glycine was then added to a final concentration of $0.125 \mathrm{M}$ and tissue was placed under vacuum for an additional 5 minutes, followed by washing several times in water. ChIP was performed on the fixed tissue using the procedure of Gendrel et al. [72]. For each ChIP reaction, we used $2 \mathrm{ug}$ of a rabbit polyclonal antibody against H3K27me3 (Millipore, catalog \#07-449). Input and ChIP DNAs were converted to Illumina sequencing libraries using the Accel-NGS 2S Plus DNA library kit according to the manufacturer's instructions (Swift Biosciences). Libraries were sequenced on an Illumina NextSeq 500 instrument using 50-nt single end reads at the University of Georgia Genomics and Bioinformatics Facility.

\section{ChIP-seq data processing}

Raw reads were mapped to the SL3.0 build of the tomato genome using Bowtie2 [73] with default parameters. Raw mapped reads were then processed using Samtools [74] to retain only those with a mapping quality score greater than or equal to 2 . Enriched regions (peaks) for H3K27me3 were then identified for each replicate using the "Findpeaks" function of the HOMER package [75]. Further analyses only considered peaks that were identified in both replicates for each genotype. For normalization and visualization, quality-filtered reads in .bam format were converted to bigwig format using the "bamcoverage" script in deepTools 2.0 [76] with a bin size of $1 \mathrm{bp}$ and RPKM normalization. Heat maps and average plots displaying ChIP-seq data were also generated using the "computeMatrix" and "plotHeatmap" functions in the deepTools 2.0 package. 


\section{Data accessibility}

RNAseq reads are available at https://www.ncbi.nlm.nih.gov/sra under project numbers PRJNA347502 (M82) and PRJNA723668 (crel-2 and crel-2).

All ChIP-seq datasets have been deposited to the NCBI GEO database and are available under accession number GSE174416.

\section{Acknowledgments:}

We thank Yuval Eshed, Alon Samach and Dani Zamir for plant material and fruitful discussions and suggestions; members of the Ori lab for continuous discussions and help; Revital Bronstein, Yuval Eshed and Zach Lippman for sharing the tomato mapping marker set.

\section{References:}

1. Mosquna A, Katz A, Decker EL, Rensing SA, Reski R, Ohad N. Regulation of stem cell maintenance by the Polycomb protein FIE has been conserved during land plant evolution. Development. 2009;136: 2433-2444. doi:10.1242/dev.035048

2. Hennig L, Derkacheva M. Diversity of Polycomb group complexes in plants: same rules, different players? Trends Genet. 2009;25: 414-423. doi:10.1016/j.tig.2009.07.002

3. Xiao J, Wagner D. Polycomb repression in the regulation of growth and development in Arabidopsis. Curr Opin Plant Biol. 2015;23: 15-24. doi:http://dx.doi.org/10.1016/j.pbi.2014.10.003

4. Geisler SJ, Paro R. Trithorax and polycomb group-dependent regulation: A tale of opposing activities. Development. Company of Biologists Ltd; 2015. pp. 2876-2887. doi:10.1242/dev.120030

5. Xiao J, Jin R, Yu X, Shen M, Wagner JD, Pai A, et al. Cis and trans determinants of epigenetic silencing by Polycomb repressive complex 2 in Arabidopsis. Nat Genet. 2017;49: 1546-1552. doi:10.1038/ng.3937 
6. Zhou Y, Wang Y, Krause K, Yang T, Dongus JA, Zhang Y, et al. Telobox motifs recruit CLF/SWN-PRC2 for H3K27me3 deposition via TRB factors in Arabidopsis. Nat Genet. 2018;50: 638-644. doi:10.1038/s41588-018-0109-9

7. De Lucia F, Crevillen P, Jones AME, Greb T, Dean C. A PHD-Polycomb Repressive Complex 2 triggers the epigenetic silencing of FLC during vernalization. Proc Natl Acad Sci. 2008;105: 16831-16836. doi:10.1073/pnas.0808687105

8. Sung S, Schmitz RJ, Amasino RM. A PHD finger protein involved in both the vernalization and photoperiod pathways in Arabidopsis. Genes Dev. 2006;20: 3244-3248. doi:10.1101/gad.1493306

9. Amasino RM, Sung S. Vernalization in Arabidopsis thaliana is mediated by the PHD finger protein VIN3. Nature. 2004;427: 159-164. doi:10.1038/nature02238.1.

10. Greb T, Dean C, Mylne JS, Crevillen P, Geraldo N, An H, et al. The PHD finger protein VRN5 functions in the epigenetic silencing of Arabidopsis FLC. Curr Biol. 2007;17: 73-78. doi:10.1016/j.cub.2006.11.052

11. Kim DH, Sung S. Coordination of the vernalization response through a VIN3 and FLC gene family regulatory network in Arabidopsis. Plant Cell. 2013;25: 454-469. doi:10.1105/tpc.112.104760

12. Kim D-H, Sung S. The Plant Homeo Domain finger protein, VIN3-LIKE 2, is necessary for photoperiod-mediated epigenetic regulation of the floral repressor, MAF5. Proc Natl Acad Sci. 2010;107: 17029-17034. doi:10.1073/pnas.1010834107

13. Zhao SQ, Hu J, Guo LB, Qian Q, Xue HW. Rice leaf inclination2, a VIN3-like protein, regulates leaf angle through modulating cell division of the collar. Cell Res. 2010;20: 935-947. doi:10.1038/cr.2010.109

14. Yoon H, Yang J, Liang W, Zhang D, An G. OsVIL2 Regulates Spikelet Development by Controlling Regulatory Genes in Oryza sativa. Front Plant Sci. 2018;9: 1-12. doi:10.3389/fpls.2018.00102 
15. Yang J, Lee S, Hang R, Kim SR, Lee YS, Cao X, et al. OsVIL2 functions with PRC2 to induce flowering by repressing O s LFL 1 in rice. Plant J. 2013;73: 566-578. doi:10.1111/tpj.12057

16. Yang J, Cho LH, Yoon J, Yoon H, Wai AH, Hong WJ, et al. Chromatin interacting factor OsVIL2 increases biomass and rice grain yield. Plant Biotechnol J. 2019;17: 178-187. doi:10.1111/pbi.12956

17. Wang J, Hu J, Qian Q, Xue HW. LC2 and OsVIL2 promote rice flowering by photoperoid-induced epigenetic silencing of OsLF. Mol Plant. 2013;6: 514527. doi:10.1093/mp/sss096

18. An Y, Guo Y, Liu C, An H. BdVIL4 regulates flowering time and branching through repressing miR156 in ambient temperature dependent way in Brachypodium distachyon. Plant Physiol Biochem. 2015;89: 92-99. doi:10.1016/j.plaphy.2015.02.013

19. Jeong HJ, Yang J, Cho LH, An G. OsVIL1 controls flowering time in rice by suppressing OsLF under short days and by inducing Ghd7 under long days. Plant Cell Rep. 2016;35: 905-920. doi:10.1007/s00299-015-1931-5

20. Fu D, Dunbar M, Dubcovsky J. Wheat VIN3-like PHD finger genes are upregulated by vernalization. Mol Genet Genomics. 2007;277: 301-313. doi:10.1007/s00438-006-0189-6

21. Almutairi ZM, Sadder MT. Cloning and expression profiling polycomb gene VERNALIZATION INSENSITIVE 3 in tomato. Biol Plant. 2014;58: 419-426. doi:10.1007/s10535-014-0421-X

22. Mohan V, Borovsky Y, Kamara I, Zemach H, Paran I. CaVIL1, a plant homeodomain gene that promotes flowering in pepper. Theor Appl Genet. 2018;131: 2639-2649. doi:10.1007/s00122-018-3179-2

23. Yoon J, Cho L-HH, Lee S, Pasriga R, Tun W, Yang J, et al. Chromatin Interacting Factor OsVIL2 Is Required for Outgrowth of Axillary Buds in Rice. Mol Cells. 2019;42: 858-868. doi:10.14348/molcells.2019.0141

24. Bar M, Ori N. Compound leaf development in model plant species. Curr Opin 
Plant Biol. 2015;23: 61-69. doi:10.1016/j.pbi.2014.10.007

25. Hagemann W, Gleissberg S. Organogenetic capacity of leaves: the significance of marginal blastozones in angiosperms. Plant Syst Evol. 1996;199: 121-152.

26. Shleizer-Burko S, Burko Y, Ben-Herzel O, Ori N. Dynamic growth program regulated by LANCEOLATE enables flexible leaf patterning. Development. 2011;138: 695-704. doi:dev.056770 [pii] 10.1242/dev.056770

27. Blein T, Hasson A, Laufs P. Leaf development: what it needs to be complex. Curr Opin Plant Biol. 2010;13: 75-82. doi:S1369-5266(09)00141-1 [pii] 10.1016/j.pbi.2009.09.017

28. Israeli A, Ben-Herzel O, Burko Y, Shwartz I, Ben-Gera H, Harpaz-Saad S, et al. Coordination of differentiation rate and local patterning in compound-leaf development. New Phytol. 2021;229: 3558-3572. doi:https://doi.org/10.1111/nph.17124

29. Israeli A, Burko Y, Shleizer-Burko S, Zelnik ID, Sela N, Hajirezaei MR, et al. Coordinating the morphogenesis-differentiation balance by tweaking the cytokinin-gibberellin equilibrium. PLOS Genet. 2021;17: e1009537. doi:10.1371/journal.pgen.1009537

30. Kierzkowski D, Runions A, Vuolo F, Huijser P, Smith RS, Tsiantis M, et al. Article A Growth-Based Framework for Leaf Shape Development and Diversity Article A Growth-Based Framework for Leaf Shape Development and Diversity. Cell. 2019;177: 1405-1418. doi:10.1016/j.cell.2019.05.011

31. Koenig D, Bayer E, Kang J, Kuhlemeier C, Sinha N. Auxin patterns Solanum lycopersicum leaf morphogenesis. Development. 2009;136: 2997-3006. doi:10.1242/dev.033811

32. Ben-Gera H, Ori N. Auxin and LANCEOLATE affect leaf shape in tomato via different developmental processes. Plant Signal Behav. 2012;7.

33. Barkoulas M, Hay A, Kougioumoutzi E, Tsiantis M. A developmental framework for dissected leaf formation in the Arabidopsis relative Cardamine hirsuta. NatGenet. 2008;40: 1136-1141. doi:10.1038/ng.189 
34. Bilsborough GD, Runions A, Barkoulas M, Jenkins HW, Hasson A, Galinha C, et al. Model for the regulation of Arabidopsis thaliana leaf margin development. Proc Natl Acad Sci U S A. 2011;108: 3424-3429. doi:1015162108 [pii] 10.1073/pnas.1015162108

35. Xiong Y, Jiao Y. The Diverse Roles of Auxin in Regulating. Plants. 2019;8: 114.

36. Israeli A, Capua Y, Shwartz I, Bar M, Efroni I, Israeli A, et al. Multiple AuxinResponse Regulators Enable Stability and Variability in Leaf Development Article Multiple Auxin-Response Regulators Enable Stability and Variability in Leaf Development. Curr Biol. 2019;29: 1746-1759.e5. doi:10.1016/j.cub.2019.04.047

37. Ben-Gera H, Dafna A, Alvarez JPP, Bar M, Mauerer M, Ori N. Auxinmediated lamina growth in tomato leaves is restricted by two parallel mechanisms. Plant J. 2016;86: 443-457. doi:10.1111/tpj.13188

38. Berger Y, Harpaz-Saad S, Brand A, Melnik H, Sirding N, Alvarez JPP, et al. The NAC-domain transcription factor GOBLET specifies leaflet boundaries in compound tomato leaves. Development. 2009;136: 823-832. doi:10.1242/dev.031625

39. Ben-Gera H, Shwartz I, Shao M-RR, Shani E, Estelle M, Ori N. ENTIRE and GOBLET promote leaflet development in tomato by modulating auxin response. Plant J. 2012;70: 903-915. doi:10.1111/j.1365-313X.2012.04939.x

40. Wang H, Jones B, Li Z, Frasse P, Delalande C, Regad F, et al. The tomato Aux/IAA transcription factor IAA9 is involved in fruit development and leaf morphogenesis. Plant Cell. 2005;17: 2676-2692. doi:10.1105/tpc.105.033415

41. Menda N, Semel Y, Peled D, Eshed Y, Zamir D. In silico screening of a saturated mutation library of tomato. Plant J. 2004;38: 861-872. doi:10.1111/j.1365-313X.2004.02088.x

42. Dengler NG, Tsukaya H. Leaf morphogenesis in dicotyledons: current issues. Int J Plant Sci. 2001;162: 459-464. 
43. Kaplan DR. Fundamental concepts of leaf morphology and morphogenesis: a contribution to the interpretation of molecular genetic mutants. Int JPlant Sci. 2001;162: 465-474.

44. Ori N, Cohen ARR, Etzioni A, Brand A, Yanai O, Shleizer S, et al. Regulation of LANCEOLATE by miR319 is required for compound-leaf development in tomato. Nat Genet. 2007;39: 787-791. doi:10.1038/ng2036

45. Bar M, Israeli A, Levy M, Gera HB, Jiménez-Gómez JM, Kouril S, et al. CLAUSA is a MYB transcription factor that promotes leaf differentiation by attenuating cytokinin signaling. Plant Cell. 2016;28: 1602-1615. doi:10.1105/tpc. 16.00211

46. Avivi Y, Lev-Yadun S, Morozova N, Libs L, Williams L, Zhao J, et al. Clausa, a tomato mutant with a wide range of phenotypic perturbations, displays a cell type-dependent expression of the homeobox gene LeT6/TKn21. Plant Physiol. 2000;124: 541-551. doi:10.1104/pp.124.2.541

47. Jasinski S, Kaur H, Tattersall A, Tsiantis M. Negative regulation of KNOX expression in tomato leaves. Planta. 2007;226: 1255-1263. doi:10.1007/s00425-007-0572-5

48. Bar M, Ben-Herzel O, Kohay H, Shtein I, Ori N. CLAUSA restricts tomato leaf morphogenesis and GOBLET expression. Plant J. 2015;83: 888-902. doi:10.1111/tpj.12936

49. Bolger A, Scossa F, Bolger ME, Lanz C, Maumus F, Tohge T, et al. The genome of the stress-tolerant wild tomato species Solanum pennellii. Nat Genet. 2014;46: 1034-1038. doi:10.1038/ng.3046

50. Moore I, Galweiler L, Grosskopf D, Schell J, Palme K. A transcription activation system for regulated gene expression in transgenic plants. Proc Natl Acad Sci USA. 1998;95: 376-381.

51. Shani E, Burko Y, Lilach B-Y, Berger Y, Amsellem Z, Goldshmidt A, et al. Stage-specific regulation of Solanum lycopersicum leaf maturation by class 1 KNOTTED1-LIKE HOMEOBOX proteins. Plant Cell. 2009;21: 3078-3092. doi:tpc.109.068148 [pii] 10.1105/tpc.109.068148 
52. Mouriz A, López-González L, Jarillo JA, Piñeiro M. PHDs govern plant development. Plant Signal Behav. 2015;10: e993253. doi:10.4161/15592324.2014.993253

53. Zhao Y, Antoniou-Kourounioti RL, Calder G, Dean C, Howard M. Temperature-dependent growth contributes to long-term cold sensing. Nature. 2020;583: 825-829. doi:10.1038/s41586-020-2485-4

54. Shalit A, Rozman A, Goldshmidt A, Alvarez JP, Bowman JL, Eshed Y, et al. The flowering hormone florigen functions as a general systemic regulator of growth and termination. Proc Natl Acad Sci U S A. 2009;106: 8392-8397. doi:10.1073/pnas.0810810106

55. Shalit-Kaneh A, Eviatar-Ribak T, Horev G, Suss N, Aloni R, Eshed Y, et al. The flowering hormone florigen accelerates secondary cell wall biogenesis to harmonize vascular maturation with reproductive development. Proc Natl Acad Sci. 2019;116: 16127-16136. doi:10.1073/pnas.1906405116

56. Lifschitz E, Ayre BG, Eshed Y. Florigen and anti-florigen â $€^{\text {“ }}$ a systemic mechanism for coordinating growth and termination in flowering plants. Front Plant Sci. 2014;5: 1-14. doi:10.3389/fpls.2014.00465

57. Navarro C, Abelenda JA, Cruz-Oró E, Cuéllar CA, Tamaki S, Silva J, et al. Control of flowering and storage organ formation in potato by FLOWERING LOCUS T. Nature. 2011;478: 119-122. doi:10.1038/nature10431

58. Cartolano M, Pieper B, Lempe J, Tattersall A, Huijser P, Tresch A, et al. Heterochrony underpins natural variation in Cardamine hirsuta leaf form. Proc Natl Acad Sci U S A. 2015;112: 10539-10544. doi:10.1073/pnas.1419791112

59. Burko Y, Shleizer-Burko S, Yanai O, Shwartz I, Zelnik ID, Jacob-Hirsch J, et al. A role for APETALA1/fruitfull transcription factors in tomato leaf development. Plant Cell. 2013;25: 2070-2083. doi:10.1105/tpc.113.113035

60. Silva GFF, Silva EM, Correa JPO, Vicente MH, Jiang N, Notini MM, et al. Tomato floral induction and flower development are orchestrated by the interplay between gibberellin and two unrelated microRNA-controlled modules. New Phytol. 2019;221: 1328-1344. doi:10.1111/nph.15492 
61. Goodrich J, Puangsomlee P, Martin M, Long D, Meyerowitz EM, Coupland G. A Polycomb-group gene regulates homeotic gene expression in Arabidopsis. Nature. 1997;386: 44-51. doi:10.1038/386044a0

62. Wang Y, Chen R. Regulation of Compound Leaf Development. Plants. 2013;3: 1-17. Available: http://www.mdpi.com/2223-7747/3/1/1

63. Shwartz I, Levy M, Ori N, Bar M. Hormones in tomato leaf development. Dev Biol. 2016;419. doi:10.1016/j.ydbio.2016.06.023

64. Kim D, Pertea G, Trapnell C, Pimentel H, Kelley R, Salzberg SL. TopHat2: Accurate alignment of transcriptomes in the presence of insertions, deletions and gene fusions. Genome Biol. 2013;14: R36. doi:10.1186/gb-2013-14-4-r36

65. Depristo MA, Banks E, Poplin R, Garimella K V., Maguire JR, Hartl C, et al. A framework for variation discovery and genotyping using next-generation DNA sequencing data. Nat Genet. 2011;43: 491-501. doi:10.1038/ng.806

66. Wang K, Li M, Hakonarson H. ANNOVAR: functional annotation of genetic variants from high-throughput sequencing data. Nucleic Acids Res. 2010;38: e164-e164. doi:10.1093/nar/gkq603

67. Kurihara D, Mizuta Y, Sato Y, Higashiyama T. ClearSee: A rapid optical clearing reagent for whole-plant fluorescence imaging. Development. 2015;142: 4168-4179. doi:10.1242/dev.127613

68. Thompson JD, Gibson TJ, Plewniak F, Jeanmougin F, Higgins DG. The CLUSTAL_X Windows Interface: Flexible Strategies for Multiple Sequence Alignment Aided by Quality Analysis Tools. Nucleic Acids Res. 1997;25: 4876-4882. doi:10.1093/nar/25.24.4876

69. Jeanmougin F, Thompson JD, Gouy M, Higgins DG, Gibson TJ. Multiple sequence alignment with Clustal X. Trends Biochem Sci. 1998;23: 403-405. doi:10.1016/S0968-0004(98)01285-7

70. Exposito-Rodriguez M, Borges AA, Borges-Perez A, Perez JA, ExpósitoRodríguez M, Borges AA, et al. Selection of internal control genes for quantitative real-time RT-PCR studies during tomato development process. 
BMC Plant Biol. 2008;8: 131. doi:1471-2229-8-131 [pii] 10.1186/1471-2229$8-131$

71. Shani E, Ben-Gera H, Shleizer-Burko S, Burko Y, Weiss D, Ori N. Cytokinin regulates compound leaf development in tomato c w. Plant Cell. 2010;22. doi:10.1105/tpc. 110.078253

72. Gendrel AV, Lippman Z, Martienssen R, Colot V. Profiling histone modification patterns in plants using genomic tiling microarrays. Nat Methods. 2005;2: 213-218. doi:10.1038/nmeth0305-213

73. Langmead B, Salzberg SL. Fast gapped-read alignment with Bowtie 2. Nat Methods. 2012;9: 357-359. doi:10.1038/nmeth.1923

74. Li H, Handsaker B, Wysoker A, Fennell T, Ruan J, Homer N, et al. The Sequence Alignment/Map format and SAMtools. Bioinformatics. 2009;25: 2078-2079. doi:10.1093/bioinformatics/btp352

75. Heinz S, Benner C, Spann N, Bertolino E, Lin YC, Laslo P, et al. Simple Combinations of Lineage-Determining Transcription Factors Prime cisRegulatory Elements Required for Macrophage and B Cell Identities. Mol Cell. 2010;38: 576-589. doi:10.1016/j.molcel.2010.05.004

76. Ramírez F, Ryan DP, Grüning B, Bhardwaj V, Kilpert F, Richter AS, et al. deepTools2: a next generation web server for deep-sequencing data analysis. Nucleic Acids Res. 2016;44: W160-W165. doi:10.1093/nar/gkw257

\section{Figure Captions:}

Fig 1. Mutating crel suppresses the $e$ simple leaf phenotype and produces very compound leaves. (A-H) Mature $5^{\text {th }}$ leaves of the indicated genotypes. A-C suppression of $e$ by crel-1; D-H: leaves of 5 different crel alleles. Scale bars: $2 \mathrm{~cm}$. (I) Early leaf development in the fifth leaf of wild type and crel-2. P4- P7 designate the developmental stages, where P4 is the forth youngest leaf primordium. Scale bars: 0.1 $\mathrm{mm}$ (P4), $0.5 \mathrm{~mm}$ (P6), $2 \mathrm{~mm}$ (P7), $2 \mathrm{~cm}$ (expanded leaf). (J) Quantification of the number of leaflets in a mature $5^{\text {th }}$ leaf of the indicated $\mathrm{crel}$ alleles, compared to the wild type. $1^{\text {st }}, 2$ nd and $3^{\text {rd }}$ represent primary, secondary and tertiary leaflets, respectivley, where primary leaflets arise from the rachis, secondary leaflets arise from primary leaflets etc. (K) Leaflet production over time by the fifth leaf (L5) of the indicated genotypes. 
Fig 2. CREL acts relatively late in leaf development to promote differentiation and blade growth. (A-F, I, J) Mature $5^{\text {th }}$ leaves. (K,L) Mature $9^{\text {th }}$ leaves. $(\mathrm{G}, \mathrm{H})$ whole plants. Scale bars: $2 \mathrm{~cm}$. FIL $>>$ gene refers to genotypes generated by the LhG4-OP transactivation system, where the gene is expressed in the FIL expression domain. The FIL promoter is expressed in leaf primordia (Shani et al., 2009); EdII-GUS is a stabilized form of $E$ fused to the GUS reporter; ARF10m is a mutant form of ARF10 that is mutated in the miR160 binding site. A-D - epistasis of $L a-2 /+$, in which differentiation is accelerated, over crel. E, F - enhancment of crel by clau, in which differentiation is delayed. G-J - enhancment of genotypes with narrow leaves due to reduced auxin response by crel. K, L - suppression of the ectopic blade growth of $F I L>>m i R 160$ by crel, similar to the effect on $e$.

Fig 3. CREL encodes a VRN5/VIL1 homolog expressed at late stages of leaf development. (A) A diagram of the CREL (Solyc05g018390) gene. The boxes indicate exons and the combining lines introns. The location of the mutation in $4 \mathrm{crel}$ alleles is indicated. (B) A phylogenetic tree of the tomato, Arabidopsis, rice and pepper VIL proteins, constructed using Clustal Omega (https://www.ebi.ac.uk/Tools/msa/clustalo/). The blue arrow points to CREL. (C) qRT-PCR analysis of CREL mRNA expression at successive developmental stages of the 5 th leaf. $m+2,3$, or 4 represents the meristem and the 2, 3, or 4 youngest leaf primordia, respectively. P4-P9 represent isolated leaf primordia at the respective developmental stage (see figure 1). Error bars represent the SE of at least three biological replicates. (D-G) Confocal images of leaf primordia of the indicated stages, expressing $p C R E L>>Y F P$, using the transactivation system, as in figure 2. P4-P7 represent the $4^{\text {th }}-7^{\text {th }}$ youngest leaf primordia, respectively. In G, a leaflet from a P7 primordium is shown. Scale bars: $0.1 \mathrm{~mm}$.

Fig 4. CREL promotes stem vasculature maturation and differentiation. (A, B) Whole plants of the indicated genotypes and ages. Scale bars: $10 \mathrm{~cm}$. (C-I) stem cross sections, dissected from the first internode of the plant (between the hypocotyl and first leaves) at the indicated times after germination and stained with Toluidine blue. Scale bars: $500 \mathrm{~mm}$. (J-P) Confocal images of stem cross sections, taken from the first internode of the plant at the indicated times after germination. Yellow arrowheads point to differentiated (WT) or undifferentiated (crel-2) xylem/ vasculature. Scale bars: 100 $\mathrm{mm}(\mathrm{J}, \mathrm{K}) ; 200 \mathrm{~mm}(\mathrm{M}) ; 500 \mathrm{~mm}(\mathrm{~L}, \mathrm{~N}-\mathrm{P})$.

Fig 5. CREL promotes root development and differentiation. (A-F) Confocal images of root cross sections of the indicated genotypes dissected from the upper part of the primary root. Scale bars: $200 \mu \mathrm{m}$ (A, B, G); $500 \mu \mathrm{m}(\mathrm{C}-\mathrm{F})$. (G) Root system volume at different times after germination, calculated with WinRhizo software. Shown are averages and SE of 3 plants $(n=3)$. Asterisks indicate statistically significant differences between crel-2 and WT, by Student's t test, ${ }^{*} \mathrm{p}<0.05$. (H) Root system length at different times after germination (DAG), calculated withWinRhizo software. Shown are averages and SE of 3 plants $(n=3)$. Asterisks indicate statistically significant differences between crel-2 and WT, by Student's t test, ${ }^{*} \mathrm{p}<0.05$. 
Fig 6. CREL promotes flowering, floral organ growth, and differentiation. (A) Flowering time, measured by number of leaves produced before flowering, of the indicated genotypes. Error bars represent the SE; $p$-values indicate differences from WT, as determined by Dunnett`s test. $\mathrm{n}=12$ (wt, crel-1) and 4 (crel-2). (B, C) Stereoscope images of mature flowers. Scale bars: $1 \mathrm{~mm}$. (D-G) Scanning electron microscope (SEM) micrographs of the indicated genotypes at 2 early developmental stages. D, F - $0.5 \mathrm{~mm}$ long stage 6 flowers; E, G - $1 \mathrm{~mm}$ long stage 11 flowers (according to (Brukhin et al., 2003). Yellow arrowheads point to normal (WT, D) and distorted (crel-2, F) young petals, and to normal (WT, E) and distorted (crel-2, F) stamens and stigma. Scale bars: $1 \mathrm{~mm}(\mathrm{E}, \mathrm{G}) ; 0.5 \mathrm{~mm}(\mathrm{D}, \mathrm{F})$.

Fig 7 . CREL mediates H3K27me3 modifications at a subset of polycomb-silenced genes. (A) Average plots and heatmaps show H3K27me3 enrichment in shoot apices of WT and crel-2 plants. The left pair of panels show the 6,762 H3K27me3-enriched sites in WT (out of a total of 13,849 sites) where the modification is significantly depleted in crel-2 mutants. The right pair of panels show 4,789 H3K27me3-enriched sites where the modification level is higher in the crel-2 mutant. The majority of these sites are normally enriched with H3K27me3 in WT and the levels become higher in crel-2. (B) qRT-PCR analysis comparing the mRNA expression of GA20oxidase 3 (GA20ox3, Solyc11g072310), ARGONAUTE 5 (AGO5, Solyc06g074730) and PECTINESTERASE (Solyc02g080200) in primordia of the fifth leaf produced by the plant at the P5 stage from wild type (WT) and crel-2 plants. These were among the genes in which $\mathrm{H} 3 \mathrm{~K} 27 \mathrm{me} 3$ was lost in crel-2 in comparison to the wild type. The bars represent the average of 3-5 biological replicates, and error bars indicate SE. Asterisks indicate statiscally significant diffrences, determined by students t-test, $* * \mathrm{P}<0.01$, $* * * \mathrm{P}<0.001$

\section{Supporting information captions:}

S1 Fig. Characterization of crel mutants. (A-D) Mature $5^{\text {th }}$ leaves of the indicated genotypes. White arrowheads point to primary and intercalary leaflets. Scale bars: $2 \mathrm{~cm}$. (E) Slower leaf production in crel-2. The Y axes shows the developmental stage (plastochron, P) of the fifth leaf produced by the plant at the indicated days after seeding. Error bars indicate SD ( $\mathrm{n}=5-11)$.

\section{S2 Fig. 35S: $C R E L$ has sublte developmental phenotype alterations when} compared with WT. (A) qRT-PCR analyzing CREL expression levels in 35S:CREL shoot apices containing the SAM and 5-6 young leaf primordia. Each repetition contained 9 or more plants. Error bars indicate SE $(n=3)$. Asterisks indicate statistically significant differences by student t-test, $* \mathrm{P}<0.05$. (B) Plant hight, measured from the cotyledons to the tip of the plants at the end of the growing season, on 15-week-old plants. Error bars represent the SE of 5 (crel-2), 3 (wt) or 5 (35S:CREL) repeats; $p$-values indicate differences from WT, as determent by Dunnett's test. (C) Flowering time of the 3 independent 35S:CREL lines in 
comparison to the wild type, measured by number of leaves produced before flowering. Error bars represent the SE of 5-12 plants; $p$-values indicate differences from WT, as determent by Dunnett`s test. (D) Total number of leaflets, measured on expanded $5^{\text {th }}$ leaves. Error bars represent the SE of 12 plants for the wild type and 6 plants for each of the 35S:CREL lines. $p$-values indicate differences from WT, as determent by Dunnett’s test.

S3 Fig. Overview of ChIP-seq datasets. Principal component analysis of input DNA and ChIP-seq samples.

S1 Table. Sequence read numbers for ChIP-seq. Two biological replicates of ChIP-seq for H3K27me3 were performed on shoot apices of WT and crell-2 plants. The table indicates for each biological replicate the number of total sequencing reads obtained, the number and percentage mapping to the tomato genome, and the total number of reads remaining after filtering for mapping quality. Reads with a MAPQ score of 2 or greater were used for further analysis.

S2 Table. Primers used in this work. 


\section{Shwartz et al. Figures and figure captions:}
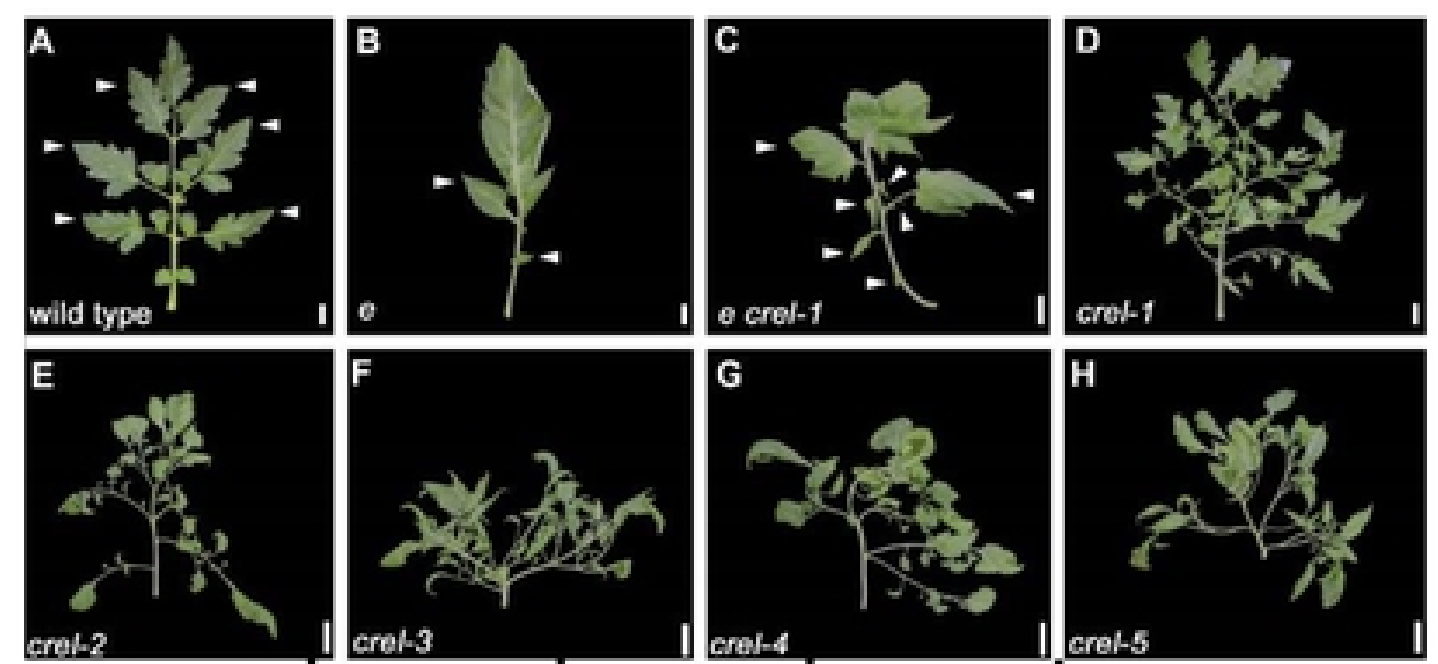

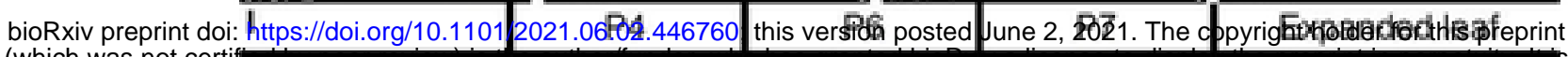
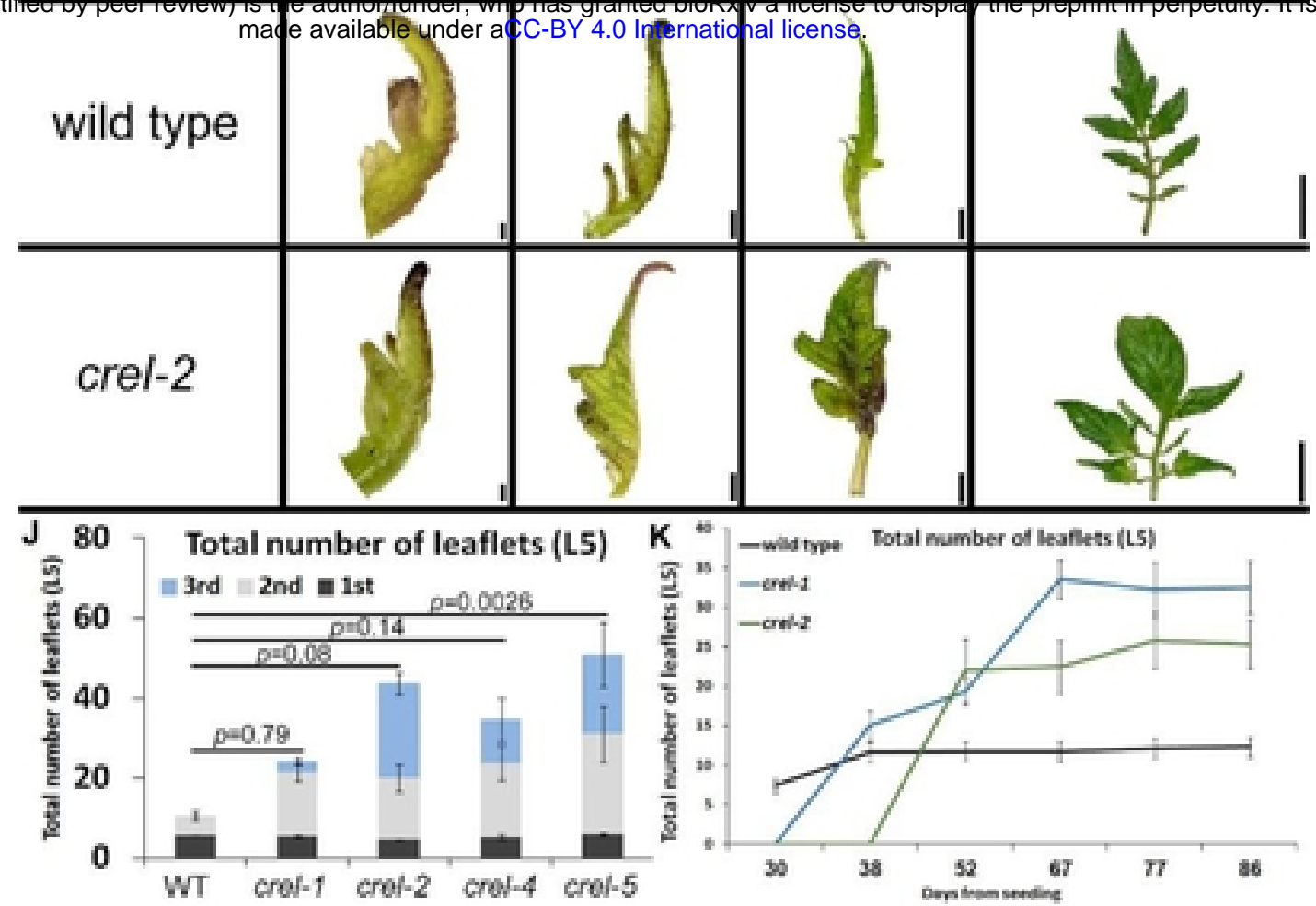

Fig 1. Mutating crel suppresses the e simple leaf phenotype and produces very compound leaves.

(A-H) Mature $5^{\text {th }}$ leaves of the indicated genotypes. A-C suppression of e by crel-1; D-H: leaves of 5 different crel alleles. Scale bars: $2 \mathrm{~cm}$. (I) Early leaf development in the fifth leaf of wild type and crel-2. P4- P7 designate the developmental stages, where P4 is the forth youngest leaf primordium. Scale bars: $0.1 \mathrm{~mm}(\mathrm{P} 4), 0.5 \mathrm{~mm}$ (P6), $2 \mathrm{~mm}$ (P7), $2 \mathrm{~cm}$ (expanded leaf). (J) Quantification of the number of leaflets in a mature $5^{\text {th }}$ leaf of the indicated crel alleles, compared to the wild type. $1^{\text {st }}, 2$ nd and $3^{\text {rd }}$ represent primary, secondary and tertiary leaflets, respectivley, where primary leaflets arise from the rachis, secondary leaflets arise from primary leaflets etc. (K) Leaflet production over time by the fifth leaf (L5) of the indicated genotypes. 

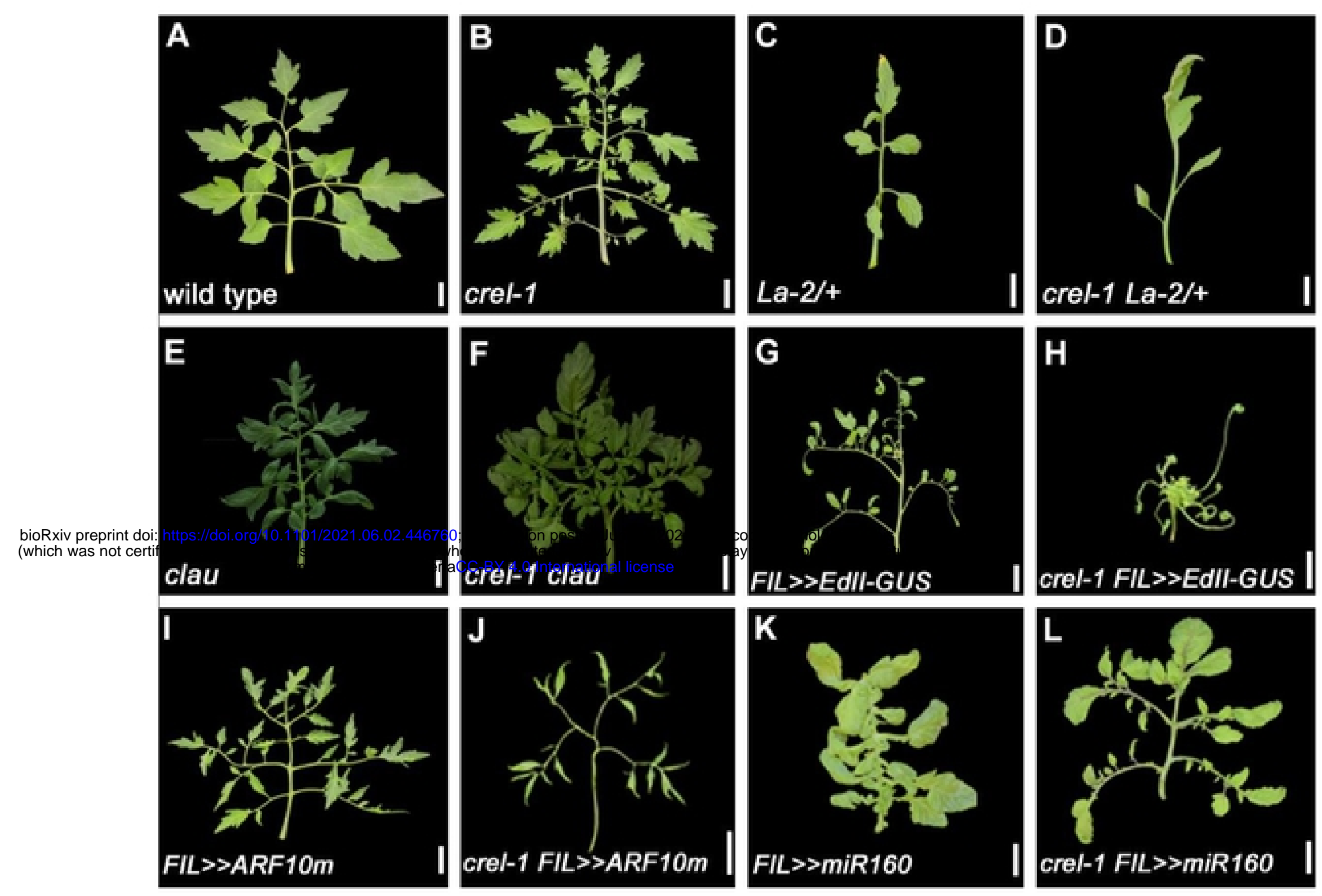

Fig 2. CREL acts relatively late in leaf development to promote differentiation and blade growth.

(A-F, I, J) Mature $5^{\text {th }}$ leaves. $(\mathrm{K}, \mathrm{L})$ Mature $9^{\text {th }}$ leaves. $(\mathrm{G}, \mathrm{H})$ whole plants. Scale bars: $2 \mathrm{~cm}$. FIL $\gg$ gene refers to genotypes generated by the LhG4-OP transactivation system, where the gene is expressed in the FIL expression domain. The FIL promoter is expressed in leaf primordia [1]; EdII-GUS is a stabilized form of E fused to the GUS reporter; ARF10m is a mutant form of ARF10 that is mutated in the miR160 binding site. A-D - epistasis of $\mathrm{La}-2 /+$, in which differentiation is accelerated, over crel. E, F enhancment of crel by clau, in which differentiation is delayed. G-J - enhancment of genotypes with narrow leaves due to reduced auxin response by crel. $\mathrm{K}, \mathrm{L}$ - suppression of the ectopic blade growth of FIL $>>$ miR160 by crel, similar to the effect on e. 
A

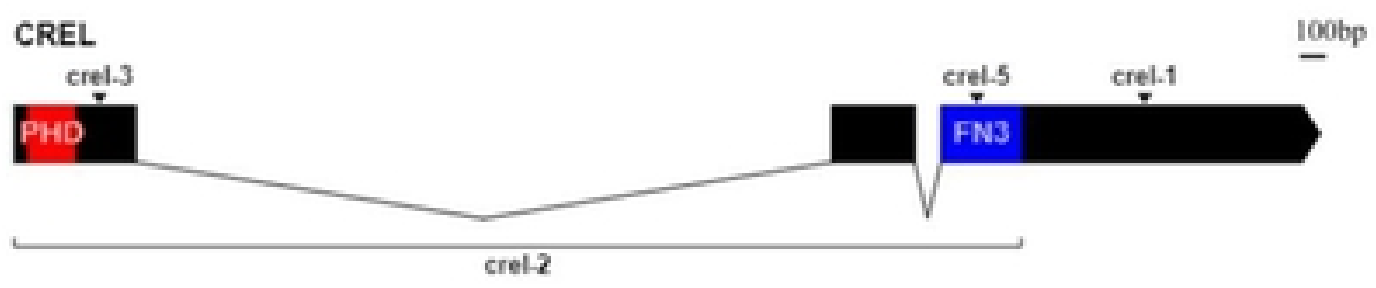

B

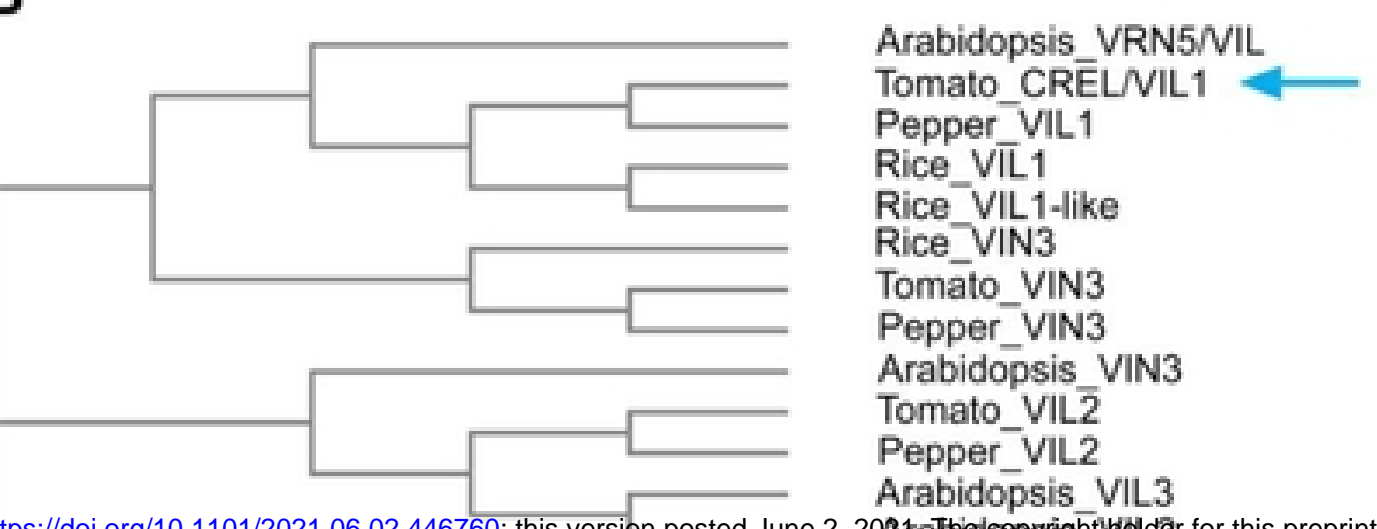

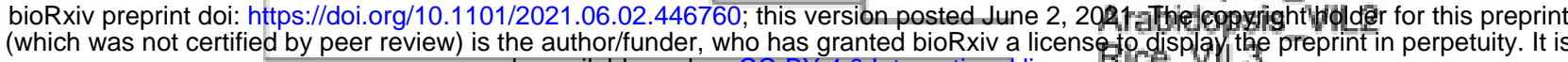

C
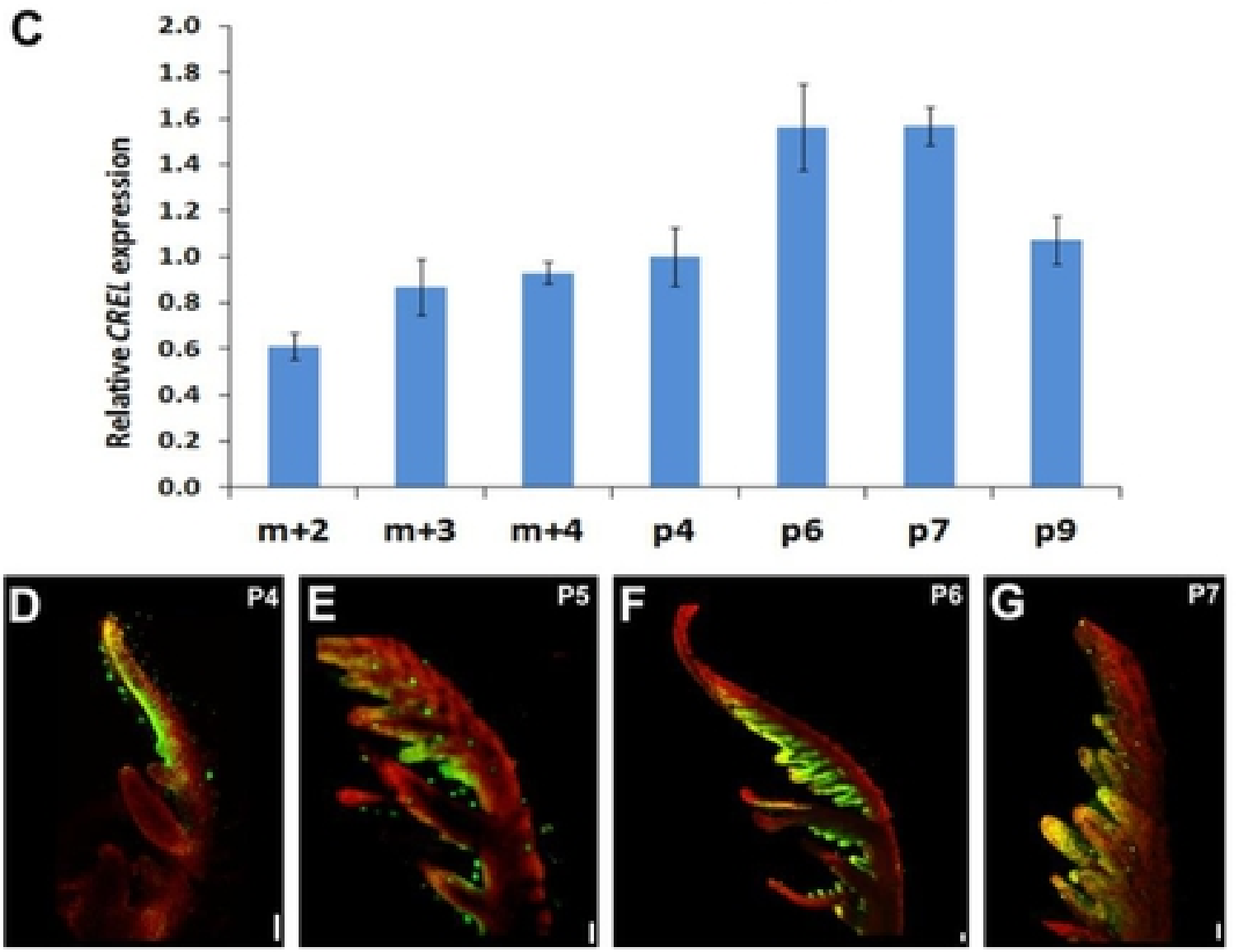

Fig 3. CREL encodes a VRN5/VIL1 homolog expressed at late stages of leaf development.

(A) A diagram of the CREL (Solyc05g018390) gene. The boxes indicate exons and the combining lines introns. The location of the mutation in $4 \mathrm{crel}$ alleles is indicated. (B) A phylogenetic tree of the tomato, Arabidopsis, rice and pepper VIL proteins, constructed using Clustal Omega (https://www.ebi.ac.uk/Tools/msa/clustalo/). The blue arrow points to CREL. (C) qRT-PCR analysis of CREL mRNA expression at successive developmental stages of the 5th leaf. $m+2,3$, or 4 represents the meristem and the 2,3 , or 4 youngest leaf primordia, respectively. P4-P9 represent isolated leaf primordia at the respective developmental stage (see figure 1). Error bars represent the SE of at least three biological replicates. (D-G) Confocal images of leaf primordia of the indicated stages, expressing pCREL $>>$ YFP, using the transactivation system, as in figure 2. P4-P7 represent the $4^{\text {th }}-7^{\text {th }}$ youngest leaf primordia, respectively. In G, a leaflet from a P7 primordium is shown. Scale bars: $0.1 \mathrm{~mm}$. 


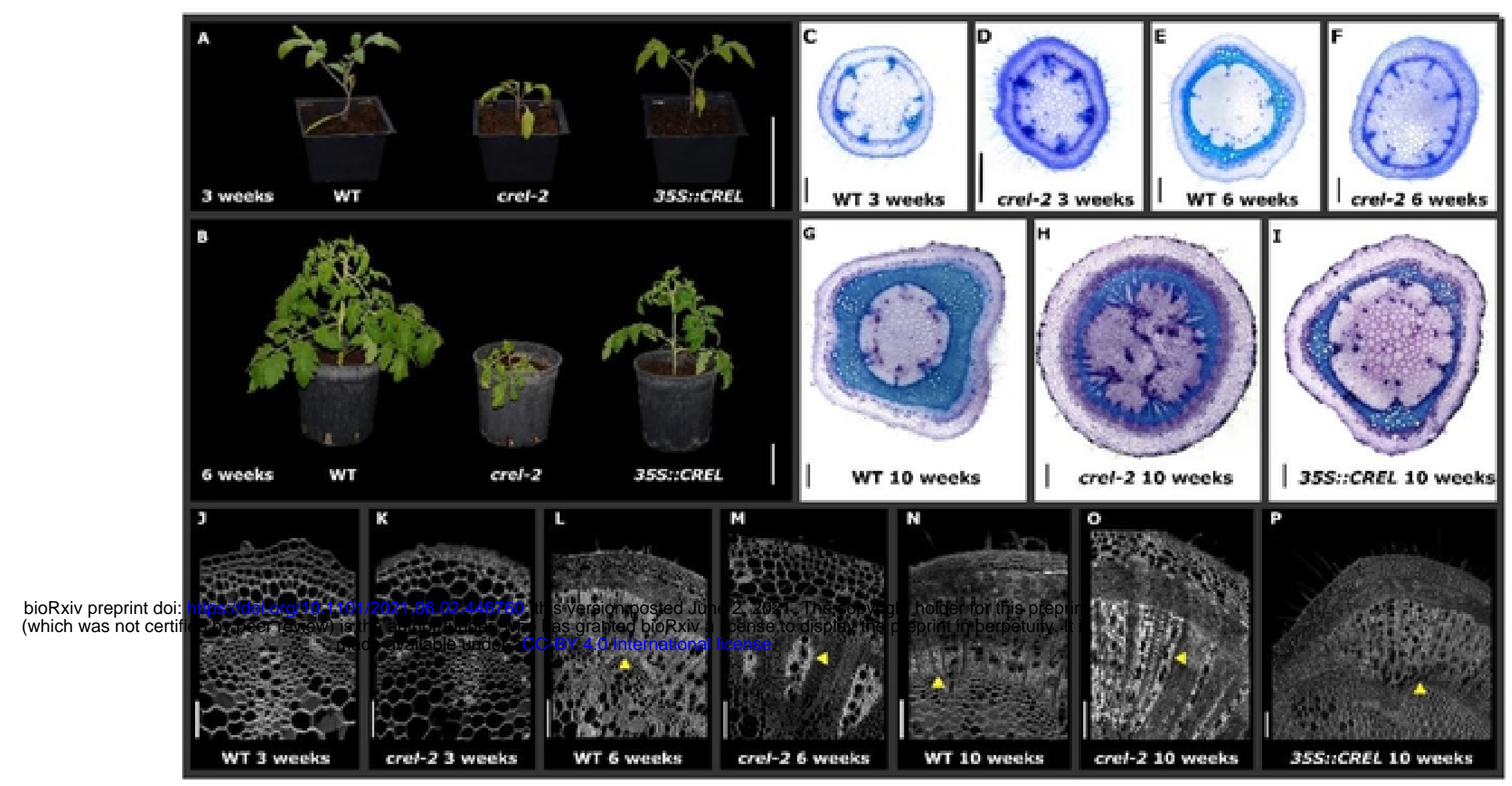

Fig 4. CREL promotes stem vasculature maturation and differentiation.

(A, B) Whole plants of the indicated genotypes and ages. Scale bars: $10 \mathrm{~cm}$. (C-I) stem cross sections, dissected from the first internode of the plant (between the hypocotyl and first leaves) at the indicated times after germination and stained with Toluidine blue. Scale bars: $500 \mathrm{~mm}$. (J-P) Confocal images of stem cross sections, taken from the first internode of the plant at the indicated times after germination. Yellow arrowheads point to differentiated (WT) or undifferentiated (crel-2) xylem/ vasculature. Scale bars: $100 \mathrm{~mm}(\mathrm{~J}, \mathrm{~K}) ; 200 \mathrm{~mm}(\mathrm{M}) ; 500 \mathrm{~mm}(\mathrm{~L}, \mathrm{~N}-\mathrm{P})$. 


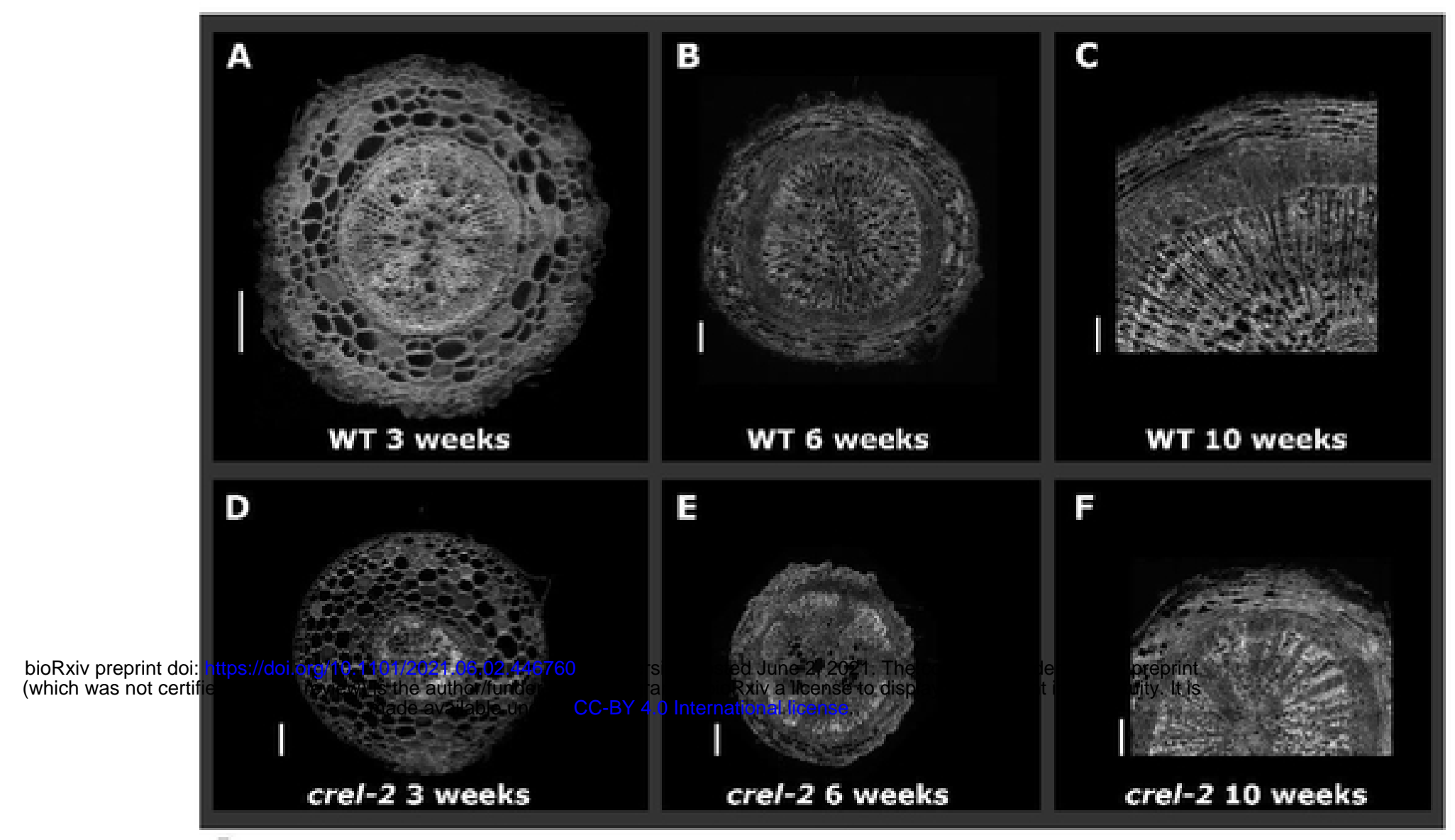

G

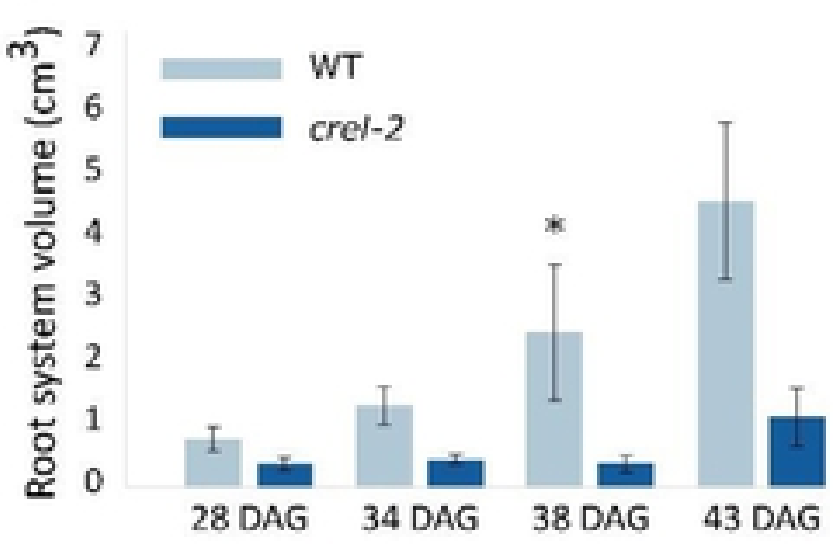

\section{H}

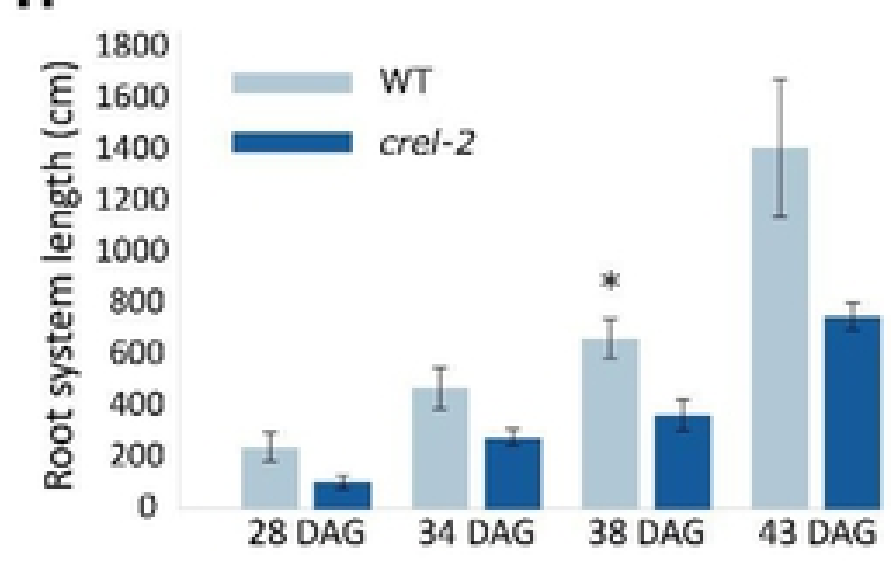

Fig 5. CREL promotes root development and differentiation.

(A-F) Confocal images of root cross sections of the indicated genotypes dissected from the upper part of the primary root. Scale bars: $200 \mu \mathrm{m}$ (A, B, G); $500 \mu \mathrm{m}(\mathrm{C}-\mathrm{F})$. (G) Root system volume at different times after germination, calculated with WinRhizo software. Shown are averages and SE of 3 plants $(n=3)$. Asterisks indicate statistically significant differences between crel-2 and WT, by Student's $t$ test, ${ }^{*} \mathrm{p}<$ 0.05 . (H) Root system length at different times after germination (DAG), calculated withWinRhizo software. Shown are averages and SE of 3 plants $(n=3)$. Asterisks indicate statistically significant differences between crel-2 and WT, by Student's $t$ test, ${ }^{*} \mathrm{p}<0.05$. 


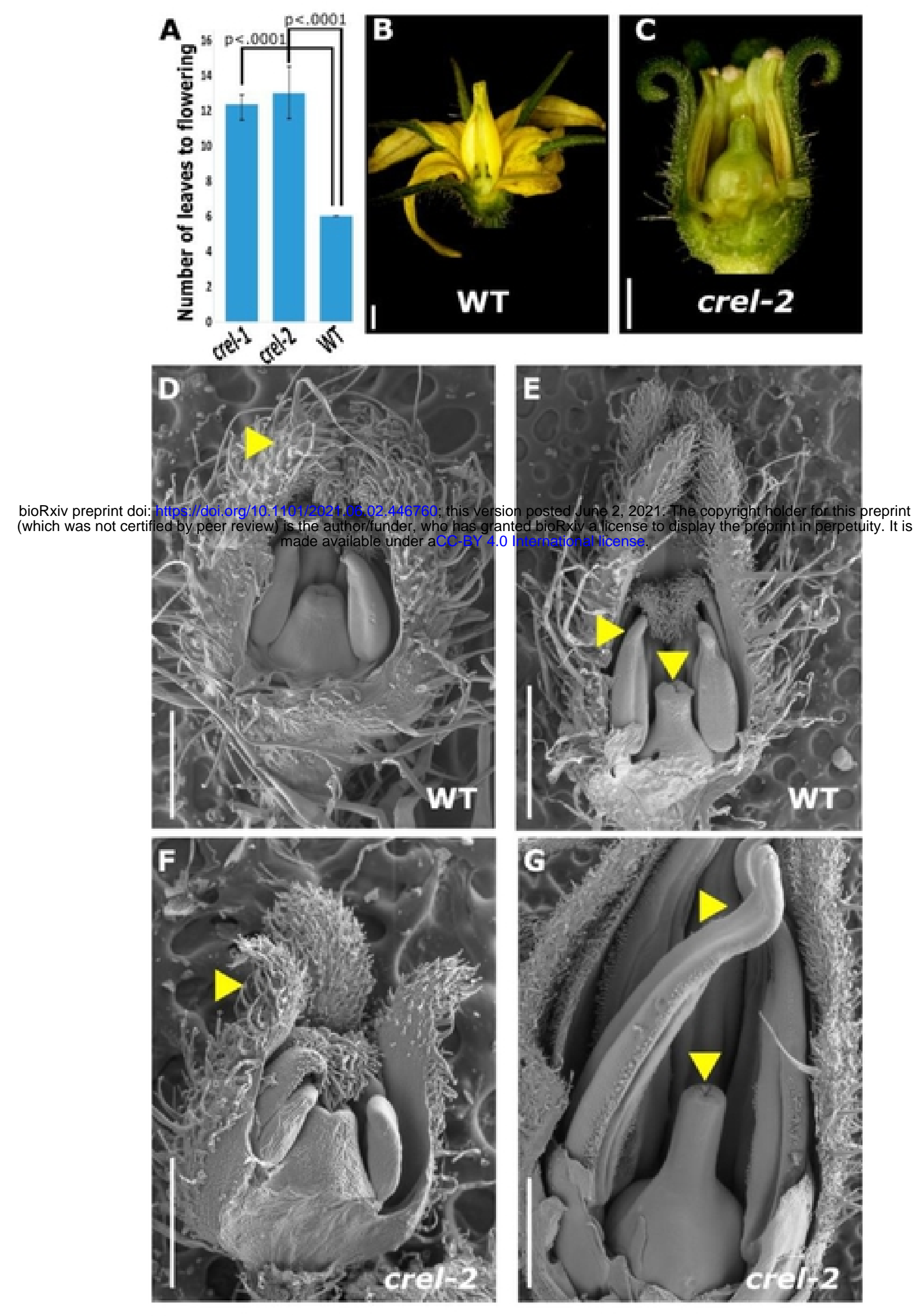

Fig 6. CREL promotes flowering, floral organ growth, and differentiation.

(A) Flowering time, measured by number of leaves produced before flowering, of the indicated genotypes. Error bars represent the SE; p-values indicate differences from WT, as determined by Dunnett's test. $\mathrm{n}=12$ (wt, crel-1) and 4 (crel-2). (B, C) Stereoscope images of mature flowers. Scale bars: $1 \mathrm{~mm}$. (D-G) Scanning electron microscope (SEM) micrographs of the indicated genotypes at 2 early developmental stages. D, F - $0.5 \mathrm{~mm}$ long stage 6 flowers; E, G - $1 \mathrm{~mm}$ long stage 11 flowers (according to [2]. Yellow arrowheads point to normal (WT, D) and distorted (crel-2, F) young petals, and to normal (WT, E) and distorted (crel-2, F) stamens and stigma. Scale bars: $1 \mathrm{~mm}(\mathrm{E}, \mathrm{G}) ; 0.5 \mathrm{~mm}(\mathrm{D}, \mathrm{F})$. 

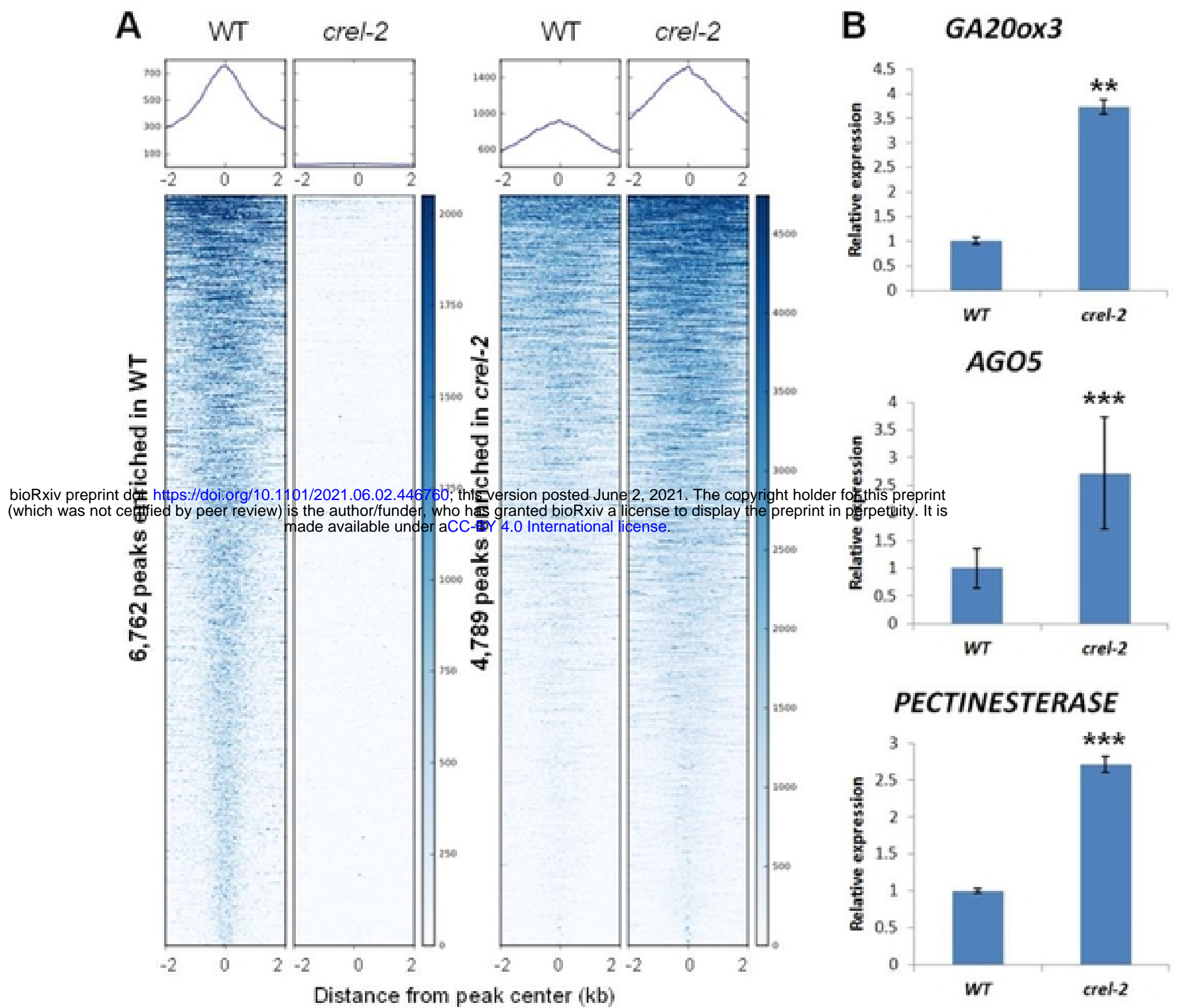

AGO5
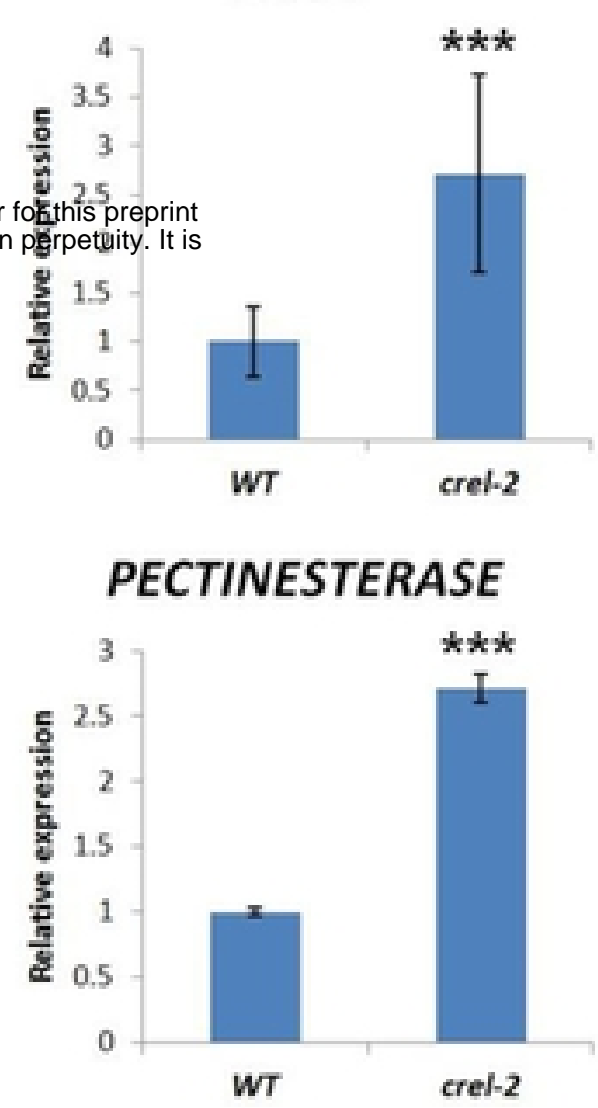

Fig 7 . CREL mediates H3K27me3 modifications at a subset of polycombsilenced genes.

(A) Average plots and heatmaps show H3K27me3 enrichment in shoot apices of WT and crel-2 plants. The left pair of panels show the 6,762 H3K27me3-enriched sites in WT (out of a total of 13,849 sites) where the modification is significantly depleted in crel-2 mutants. The right pair of panels show 4,789 H3K27me3-enriched sites where the modification level is higher in the crel-2 mutant. The majority of these sites are normally enriched with $\mathrm{H} 3 \mathrm{~K} 27 \mathrm{me} 3$ in WT and the levels become higher in crel-2. (B) qRT-PCR analysis comparing the mRNA expression of GA20oxidase 3 (GA20ox3, Solyc11g072310), ARGONAUTE 5 (AGO5, Solyc06g074730) and PECTINESTERASE (Solyc02g080200) in primordia of the fifth leaf produced by the plant at the P5 stage from wild type (WT) and crel-2 plants. These were among the genes in which $\mathrm{H} 3 \mathrm{~K} 27 \mathrm{me} 3$ was lost in crel-2 in comparison to the wild type. The bars represent the average of 3-5 biological replicates, and error bars indicate SE. Asterisks indicate statiscally significant diffrences, determined by students t-test, $* * \mathrm{P}<0.01$, $* * * \mathrm{P}<0.001$. 
9 or more plants. Error bars indicate SE $(n=3)$. Asterisks indicate statistically significant differences by student t-test, $* \mathrm{P}<0.05$. (B) Plant hight, measured from the cotyledons to the tip of the plants at the end of the growing season, on 15-week-old plants. Error bars represent the SE of 5 (crel-2), 3 (wt) or 5 (35S:CREL) repeats; pvalues indicate differences from WT, as determent by Dunnett's test. (C) Flowering time of the 3 independent 35S:CREL lines in comparison to the wild type, measured by number of leaves produced before flowering. Error bars represent the SE of 5-12 plants; p-values indicate differences from WT, as determent by Dunnett's test. (D) Total number of leaflets, measured on expanded $5^{\text {th }}$ leaves. Error bars represent the

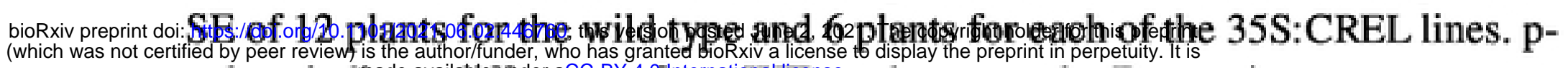

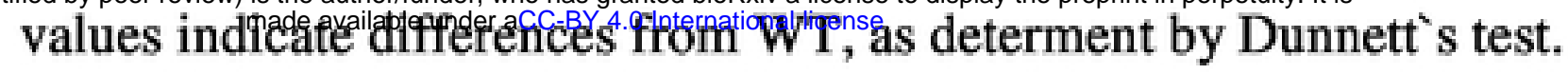




\section{References:}

1. Shani E, Burko Y, Lilach B-Y, Berger Y, Amsellem Z, Goldshmidt A, et al. Stage-specific regulation of Solanum lycopersicum leaf maturation by class 1 KNOTTED1-LIKE HOMEOBOX proteins. Plant Cell. 2009;21: 3078-3092. doi:tpc.109.068148 [pii] 10.1105/tpc.109.068148

2. Brukhin V, Hernould M, Gonzalez N, Chevalier C, Mouras A. Flower development schedule in tomato Lycopersicon esculentum cv. sweet cherry. Sex Plant Reprod. 2003;15: 311-320. doi:10.1007/s00497-003-0167-7 